\title{
Atmospheric Hydrogenation of Esters Catalyzed by PNP-Ruthenium Complexes with an $\mathbf{N}$-Heterocyclic Carbene Ligand
}

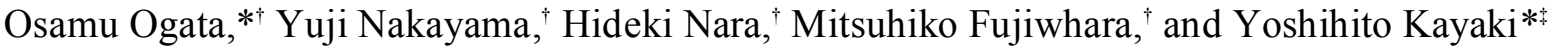 \\ ${ }^{\dagger}$ Corporate Research and Development Division, Takasago International Corporation, 1-4-11 Nishi-Yawata, Hiratsuka, \\ Kanagawa 254-0073, Japan \\ ${ }^{\ddagger}$ Department of Chemical Science and Engineering, School of Materials and Chemical Technology, Tokyo Institute of Tech- \\ nology, 2-12-1-E4-1 O-okayama, Meguro-ku, Tokyo 152-8552, Japan
}

\section{Table of Contents}

General Information

Synthetic Procedures

Hydrogenation of Esters

X-ray Structure Determination for 7.

${ }^{1} \mathrm{H},{ }^{31} \mathrm{P},{ }^{13} \mathrm{C} \mathrm{NMR}$, and HRMS Spectra 


\section{General Information}

All the oxygen and moisture-sensitive compounds were prepared and handled under a nitrogen atmosphere using standard Schlenk techniques. Dehydrated solvents $(\mathrm{MeOH}, \mathrm{EtOH}, 2$-propanol, THF, Toluene, and acetonitrile) were obtained from commercial suppliers (Aldrich, TCI, and Wako) and used after vacuum degassing. Dichloromethane- $\mathrm{d}_{2}$ and a THF solution of $\mathrm{KO}^{t} \mathrm{Bu}(1 \mathrm{M})$ were purchased from Aldrich. A MeOH solution of $\mathrm{NaOMe}(1 \mathrm{M})$ was prepared by treatment of sodium metal with anhydrous $\mathrm{MeOH}$. Aqueous $10 \%$ and $15 \% \mathrm{NaOH}$ solutions were prepared by dissolving pellets (reagent grade, Wako) in distilled water. Imidazolium salts such as 1,3-dimethylimidazolium chloride (2c, TCI), 1,3-diisopropylimidazolium chloride (2d, Aldrich), 1,3-diisopropylimidazolium tetrafluoroborate (2e, Aldrich), 1,3-dicyclohexylimidazolium tetrafluoroborate (2f, Aldrich), 1,3-di-tert-butylimidazolium tetrafluoroborate (2g, Aldrich), 1,3-bis(2,4,6-trimethylphenyl)imidazolium chloride (2h, Aldrich), 1,3-diisopropylimidazolinium tetrafluoroborate (2ii, TCI), and 1,3,4,5-tetramethylimidazolium chloride (2j. Innovative Valency) were used as delivered. Other NHC precursors such as silver(I) complex of 1,3-dimethyl-imidazol-2-ylidene (2a) $)^{1}, 1,3$-dimethylimidazolium2-carboxylate (2b $)^{2}$, and 1,3-dimethylbenzimidazolium iodide $(\mathbf{2 k})^{3}$ were synthesized according to the literature procedures. $\left[\mathrm{RuCl}_{2}(p \text {-cymene) }]_{2}\right.$, Milstein's catalyst (4) and Gusev's catalyst (5) was purchased from Aldrich. $\left(\mathrm{Ph}_{2} \mathrm{PCH}_{2} \mathrm{CH}_{2}\right)_{2} \mathrm{NH}$ and $\left({ }^{i} \mathrm{Pr}_{2} \mathrm{PCH}_{2} \mathrm{CH}_{2}\right)_{2} \mathrm{NH}$ were synthesized according to literature. ${ }^{4,5}\left(\mathrm{Ar}_{2} \mathrm{PCH}_{2} \mathrm{CH}_{2}\right)_{2} \mathrm{NH}(\mathrm{Ar}=3,5-$ bis(trifluoromethyl)phenyl) and $\left(\mathrm{Cy}_{2} \mathrm{PCH}_{2} \mathrm{CH}_{2}\right)_{2} \mathrm{NH}$ were synthesized in a similar method. Ru-MACHO was synthesized following the literature procedure. ${ }^{6}$ All esters and alcohols were obtained from the commercial source (Aldrich, TCI, or Wako). Esters were used after distillation or drying over $4 \AA$ molecular sieves as needed. Decane and tridecane was purchased from TCI. ${ }^{1} \mathrm{H}$ NMR spectra were obtained at 300 and $400-\mathrm{MHz}$ on MERCURY $300-\mathrm{C} / \mathrm{H}$ (VARIAN) and 400MR/DD2 (Agilent) spectrometers, respectively. ${ }^{31} \mathrm{P}$ NMR spectra were obtained at 121 and $161-\mathrm{MHz}$ on MERCURY 300-C/H (VARIAN) and 400MR/DD2 (Agilent) spectrometers, respectively. ${ }^{13} \mathrm{C}$ NMR spectra were obtained at $125-\mathrm{MHz}$ on an Avance III 500 (Bruker) spectrometer. Chemical shifts were referenced to a $\mathrm{CH}_{2} \mathrm{Cl}_{2}$ signal (5.32 ppm) by using the signals of $\mathrm{CH}_{2} \mathrm{Cl}_{2}-\mathrm{d}$ for ${ }^{1} \mathrm{H},{ }^{13} \mathrm{C} \mathrm{NMR}$, and an external $\mathrm{H}_{3} \mathrm{PO}_{4}$ signal $(0 \mathrm{ppm})$ for ${ }^{31} \mathrm{P} \mathrm{NMR}$. GC analysis was carried out with a GL-Sciences GC-4000 instrument equipped with a DB-WAX column (J\&W Scientific) using decane or tridecane as the internal standard. High resolution mass spectra were obtained on a LCMASS-IT-TOF spectrometer (Shimadzu) for ESI and APCI. Elemental analyses were carried out using a PE2400 Series II CHNS/O Analyzer (PerkinElmer).

\section{Synthetic Procedures Synthesis of $\left[\mathrm{RuCl}_{2}(\mathrm{PNP})\right]_{2}(1)$.}

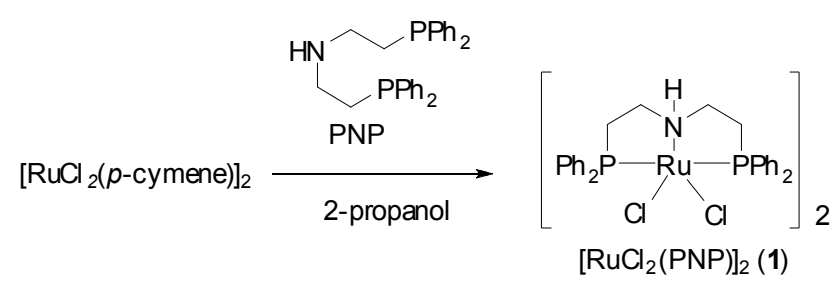

To a $20 \mathrm{~mL}$ round-bottomed flask were added bis[2-(diphenylphosphino)ethyl]amine hydrochloride (PNP·HCl, $189 \mathrm{mg}$, $0.40 \mathrm{mmol}), 10 \% \mathrm{NaOH}$ aq. $(2 \mathrm{~mL})$, and toluene $(4 \mathrm{~mL})$. The mixture was stirred at room temperature until the solids dissolved. After separation of the solution, the organic layer was washed with $\mathrm{H}_{2} \mathrm{O}(2 \mathrm{~mL} \times 2)$, dried over $\mathrm{Na}_{2} \mathrm{SO}_{4}$, and then concentrated under reduced pressure. The resulting PNP was diluted with $3 \mathrm{~mL}$ of 2 -propanol.

To a sealed reaction container were added $\left[\mathrm{RuCl}_{2}(p \text {-cymene })\right]_{2}(109 \mathrm{mg}, 0.36 \mathrm{mmol} / \mathrm{Ru})$ and the PNP solution under a nitrogen atmosphere. The reaction mixture was stirred at $120{ }^{\circ} \mathrm{C}$ for 2 hours and $150{ }^{\circ} \mathrm{C}$ for 2 hours, and then cooled to room temperature. The precipitated crystals were separated by filtration, washed with $1 \mathrm{~mL}$ of 2 -propanol and dried under reduced pressure to give $176.1 \mathrm{mg}(0.29 \mathrm{mmol} / \mathrm{Ru}, 81 \%)$ of $\left[\mathrm{RuCl}_{2}(\mathrm{PNP})\right]_{2}(\mathbf{1})$ as an orange solid.

${ }^{1} \mathrm{H}$ NMR $\left(400 \mathrm{MHz}, \mathrm{CD}_{2} \mathrm{Cl}_{2}\right): \delta 2.05-2.30(\mathrm{~m}, 4 \mathrm{H}), 2.65-2.80(\mathrm{~m}, 4 \mathrm{H}), 2.80-3.00(\mathrm{~m}, 4 \mathrm{H}), 3.30-3.50(\mathrm{~m}, 4 \mathrm{H})$, $6.68-6.80(\mathrm{~m}, 10 \mathrm{H}), 6.82-6.92(\mathrm{~m}, 4 \mathrm{H}), 7.00-7.18(\mathrm{~m}, 20 \mathrm{H}), 7.70-7.90(\mathrm{~m}, 8 \mathrm{H}) .{ }^{31} \mathrm{P}$ NMR $(161 \mathrm{MHz}$, $\left.\mathrm{CD}_{2} \mathrm{Cl}_{2}\right): \delta$ 64.2. ${ }^{13} \mathrm{C}$ NMR $\left(125 \mathrm{MHz}, \mathrm{CD}_{2} \mathrm{Cl}_{2}\right): \delta 29.99(\mathrm{~m}), 53.51(\mathrm{~s}), 127.24(\mathrm{~m}), 128.04(\mathrm{~m}), 128.45(\mathrm{~s}), 128.81(\mathrm{~s})$, $130.63(\mathrm{t}, J=3.8 \mathrm{~Hz}), 132.59(\mathrm{t}, J=4.4 \mathrm{~Hz}), 135.36(\mathrm{t}, J=20.6 \mathrm{~Hz}), 136.56(\mathrm{t}, J=21.9 \mathrm{~Hz})$. HRMS (ESI, $m / z)$ calcd for $\mathrm{C}_{56} \mathrm{H}_{58} \mathrm{~N}_{2} \mathrm{P}_{4} \mathrm{Cl}_{3} \mathrm{Ru}_{2}\left([\mathrm{M}-\mathrm{Cl}]^{+}\right): 1191.0710$. Found: 1191.0703 . 


\section{Synthesis of $\mathrm{RuCl}_{2}(p$-cymene)(NHC).}

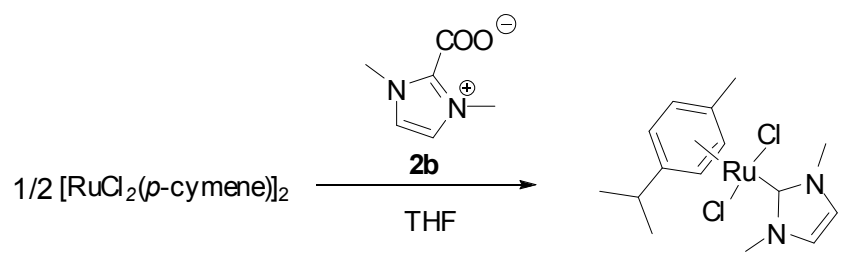

Following the reported procedure, ${ }^{7}\left[\mathrm{RuCl}_{2}(p \text {-cymene })\right]_{2}(3.85 \mathrm{~g}, 12.6 \mathrm{mmol} / \mathrm{Ru}), \mathbf{2 b}(1.83 \mathrm{~g}, 13.1 \mathrm{mmol})$, and dry THF $(25 \mathrm{~mL})$ were added to a $300 \mathrm{~mL}$ four-neck round-bottomed flask under a nitrogen atmosphere. The reaction mixture was stirred at reflux temperature for 4 hours. After cooling to room temperature, $15 \mathrm{~mL}$ of the THF was removed under reduced pressure. After addition of 2-propanol $(10 \mathrm{~mL})$, the resulting mixture was refluxed for $10 \mathrm{~min}$ and then cooled to room temperature. Subsequent addition of hexane $(5 \mathrm{~mL})$ with stirring the mixture in the ice bath for 1 hour resulted in formation of an orange precipitate. The precipitated crystals were separated by filtration and washed with $5 \mathrm{~mL}$ of hexane and dried under reduced pressure to give $4.20 \mathrm{~g}(10.44 \mathrm{mmol}, 83 \%)$ of $\mathrm{RuCl}_{2}(p$-cymene)(NHC) as an orange solid.

${ }^{1} \mathrm{H}$ NMR (300 MHz, $\mathrm{CD}_{2} \mathrm{Cl}_{2}$ ): $\delta 1.24(\mathrm{~d}, J=6.9 \mathrm{~Hz}, 6 \mathrm{H}), 1.98(\mathrm{~s}, 3 \mathrm{H}), 2.92$ (quint, $\left.J=6.9 \mathrm{~Hz}, 1 \mathrm{H}\right), 3.95(\mathrm{~s}, 6 \mathrm{H})$, $5.06(\mathrm{~d}, J=6.0 \mathrm{~Hz}, 2 \mathrm{H}), 5.39(\mathrm{~d}, J=6.0 \mathrm{~Hz}, 2 \mathrm{H}), 7.02(\mathrm{~s}, 2 \mathrm{H}) .{ }^{13} \mathrm{C}$ NMR $\left(125 \mathrm{MHz}, \mathrm{CD}_{2} \mathrm{Cl}_{2}\right): \delta 18.68,22.39,31.07$, 39.67, 82.14, 85.98, 99.05, 109.67, 124.03, 174.24. HRMS (ESI, $m / z)$ calcd for $\mathrm{C}_{15} \mathrm{H}_{22} \mathrm{~N}_{2} \mathrm{ClRu}\left([\mathrm{M}-\mathrm{Cl}]^{+}\right): 367.0510$. Found: 367.0493 .

\section{Synthesis of Ruthenium Complex 6.}
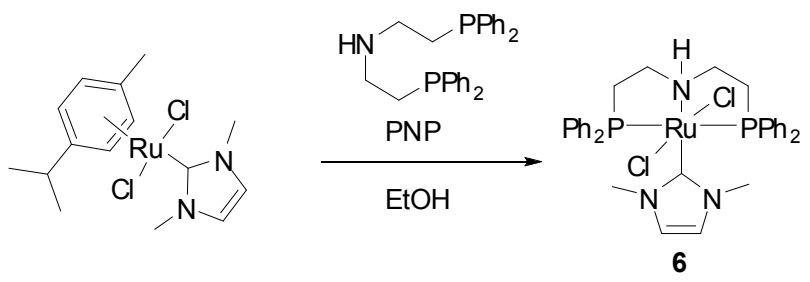

To a $50 \mathrm{~mL}$ round-bottomed flask were added PNP. $\mathrm{HCl}(724 \mathrm{mg}, 1.51 \mathrm{mmol}), 15 \% \mathrm{NaOH}$ aq. $(10 \mathrm{~mL})$, and toluene $(10 \mathrm{~mL})$. The mixture was stirred at room temperature until the solids dissolved. After separation of the solution, the organic layer was washed with $\mathrm{H}_{2} \mathrm{O}(5 \mathrm{~mL} \times 2)$, dried over $\mathrm{Na}_{2} \mathrm{SO}_{4}$, and then concentrated under reduced pressure. The resulting PNP was diluted with $3 \mathrm{~mL}$ of ethanol.

To a $20 \mathrm{~mL}$ Schlenk flask were added $\mathrm{RuCl}_{2}(p$-cymene)(NHC) $(609.3 \mathrm{mg}, 1.51 \mathrm{mmol})$ and the PNP solution under a nitrogen atmosphere. The reaction mixture was stirred at $70{ }^{\circ} \mathrm{C}$ for 2 hours, and then cooled to room temperature. After removal of ethanol under reduced pressure, $3 \mathrm{~mL}$ of hexane was added to the mixture. The resulting precipitates were separated by filtration, washed with hexane $(2 \mathrm{~mL} \times 2)$ and ethyl acetate $(2 \mathrm{~mL})$, and then dried under reduced pressure to give $916.8 \mathrm{mg}(1.29 \mathrm{mmol}, 85 \%)$ of $\mathbf{6}$ as a pale yellow solid.

${ }^{1} \mathrm{H}$ NMR $\left(400 \mathrm{MHz}, \mathrm{CD}_{2} \mathrm{Cl}_{2}\right): \delta 2.60-2.80(\mathrm{~m}, 2 \mathrm{H}), 2.95-3.15(\mathrm{~m}, 2 \mathrm{H}), 3.07(\mathrm{~s}, 3 \mathrm{H}), 3.21(\mathrm{~s}, 3 \mathrm{H}), 3.19-3.50(\mathrm{~m}$, $4 \mathrm{H}), 4.20-4.40(\mathrm{~m}, 1 \mathrm{H}), 6.78-6.86(\mathrm{~m}, 2 \mathrm{H}), 7.20-7.50(\mathrm{~m}, 20 \mathrm{H}) .{ }^{31} \mathrm{P}$ NMR $\left(161 \mathrm{MHz}, \mathrm{CD}_{2} \mathrm{Cl}_{2}\right): \delta 42.8 .{ }^{13} \mathrm{C}$ NMR (125 MHz, $\left.\mathrm{CD}_{2} \mathrm{Cl}_{2}\right): \delta 34.17(\mathrm{t}, J=10.6 \mathrm{~Hz}), 39.40(\mathrm{~s}), 40.14(\mathrm{~s}), 50.19(\mathrm{t}, J=3.8 \mathrm{~Hz}), 122.84(\mathrm{~s}), 122.90(\mathrm{~s})$, $127.70(\mathrm{t}, J=4.4 \mathrm{~Hz}), 127.83(\mathrm{t}, J=4.4 \mathrm{~Hz}), 128.95(\mathrm{~s}), 129.06(\mathrm{~s}), 133.86(\mathrm{t}, J=5.0 \mathrm{~Hz}), 137.38(\mathrm{t}, J=16.9 \mathrm{~Hz})$, 138.37 (t, $J=18.1 \mathrm{~Hz}$ ), 183.36 (t, $J=11.3 \mathrm{~Hz}$ ). Anal. calcd for $\mathrm{C}_{33} \mathrm{H}_{37} \mathrm{~N}_{3} \mathrm{P}_{2} \mathrm{Cl}_{2} \mathrm{Ru}: \mathrm{C}, 55.85 ; \mathrm{H}, 5.26 ; \mathrm{N}, 5.92$. Found: C, 55.46; H, 5.44; N, 5.78. HRMS (ESI, $m / z)$ calcd for $\mathrm{C}_{33} \mathrm{H}_{37} \mathrm{~N}_{3} \mathrm{P}_{2} \mathrm{ClRu}\left([\mathrm{M}-\mathrm{Cl}]^{+}\right)$: 674.1195 , Found: 674.1199 . 


\section{Synthesis of Ruthenium Complex 7.}
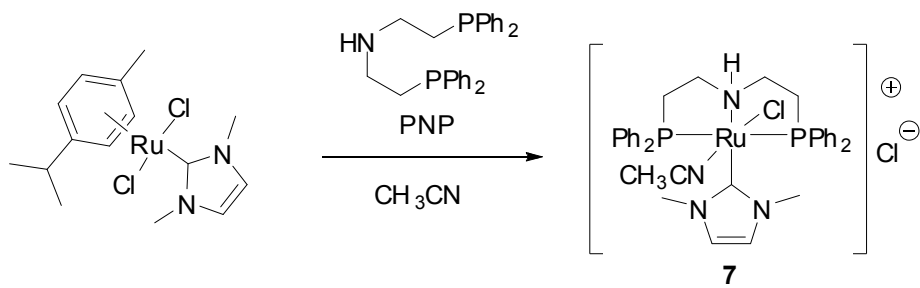

To a $50 \mathrm{~mL}$ round-bottomed flask were added $\mathrm{PNP} \cdot \mathrm{HCl}(375 \mathrm{mg}, 0.78 \mathrm{mmol}), 10 \% \mathrm{NaOH}$ aq. (10 mL), and toluene $(10 \mathrm{~mL})$. The mixture was stirred at room temperature until the solids dissolved. After separation of the solution, the organic layer was washed with $\mathrm{H}_{2} \mathrm{O}(5 \mathrm{~mL} \times 2)$, dried over $\mathrm{Na}_{2} \mathrm{SO}_{4}$, and then concentrated under reduced pressure. The resulting PNP was diluted with $5 \mathrm{~mL}$ of $\mathrm{CH}_{3} \mathrm{CN}$.

To a $50 \mathrm{~mL}$ four-necked round-bottomed flask was added a mixture of $\mathrm{RuCl}_{2}$ ( $p$-cymene)(NHC) (287 mg, $\left.0.71 \mathrm{mmol}\right)$, the PNP solution, and $\mathrm{CH}_{3} \mathrm{CN}(25 \mathrm{~mL})$ under a nitrogen atmosphere. The reaction mixture was stirred at reflux for 2 hours, and then cooled to room temperature. The precipitated crystals were separated by filtration and dried under reduced pressure to give $161.0 \mathrm{mg}(0.21 \mathrm{mmol}, 30 \%)$ of 7 as a yellow solid. Recrystallization by slow diffusion of diisopropyl ether into a dichloromethane solution of 7 afforded yellow crystals suitable for X-ray crystallography.

${ }^{1} \mathrm{H}$ NMR (300 MHz, $\left.\mathrm{CD}_{2} \mathrm{Cl}_{2}\right): \delta 1.79(\mathrm{~s}, 3 \mathrm{H}), 2.71(\mathrm{~s}, 3 \mathrm{H}), 2.90-3.50(\mathrm{~m}, 12 \mathrm{H}), 6.80-6.95(\mathrm{~m}, 2 \mathrm{H}), 7.28-7.50(\mathrm{~m}, 20$ H). ${ }^{31} \mathrm{P}$ NMR (121 MHz, $\left.\mathrm{CD}_{2} \mathrm{Cl}_{2}\right): \delta 43.6 .{ }^{13} \mathrm{C}$ NMR $\left(125 \mathrm{MHz}, \mathrm{CD}_{2} \mathrm{Cl}_{2}\right): \delta 5.41(\mathrm{~s}), 34.57(\mathrm{t}, J=11.9 \mathrm{~Hz}), 38.41(\mathrm{~s})$, 40.25 (s), 49.82 (s), 123.08 (s), 123.86 (s), $126.42(\mathrm{~s}), 128.33(\mathrm{t}, J=3.8 \mathrm{~Hz}), 128.61(\mathrm{t}, J=3.8 \mathrm{~Hz}), 129.66$ (s), 129.96 $(\mathrm{s}), 133.14(\mathrm{t}, J=5.0 \mathrm{~Hz}), 133.90(\mathrm{t}, J=5.0 \mathrm{~Hz}), 135.26(\mathrm{t}, J=18.8 \mathrm{~Hz}), 138.69(\mathrm{t}, J=18.8 \mathrm{~Hz}), 181.21(\mathrm{t}, J=10.6$ Hz). Anal. calcd for $\mathrm{C}_{35} \mathrm{H}_{40} \mathrm{~N}_{4} \mathrm{P}_{2} \mathrm{Cl}_{2} \mathrm{Ru}$ : C, 56.00; H, 5.37; N, 7.47. Found: C, 55.88; H, 5.49; N, 7.52. HRMS (ESI, $m / z)$ calcd for $\mathrm{C}_{35} \mathrm{H}_{40} \mathrm{~N}_{4} \mathrm{P}_{2} \mathrm{ClRu}\left([\mathrm{M}-\mathrm{Cl}]^{+}\right): 715.1461$. Found: 715.1433.

\section{Synthesis of Ruthenium Complex 8.}
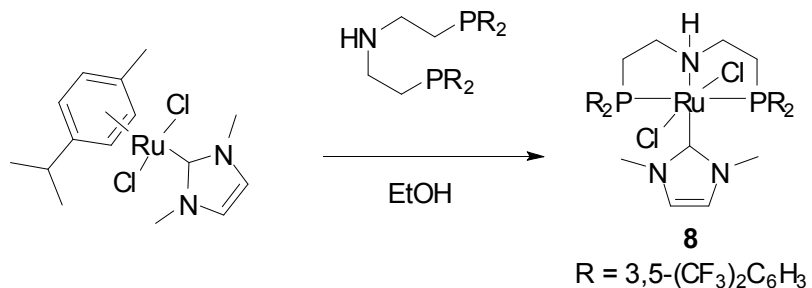

To a $20 \mathrm{~mL}$ Schlenk flask were added $\mathrm{RuCl}_{2}(p$-cymene $)(\mathrm{NHC}) \quad(700 \mathrm{mg}, 1.74 \mathrm{mmol})$, bis[2-[bis $\{3,5-$ bis(trifluoromethyl)phenyl]phosphanyl $\}$ ethyl]amine $(1.71 \mathrm{~g}, 1.74 \mathrm{mmol})$, toluene $(15.5 \mathrm{~mL})$, and ethanol $(1.7 \mathrm{~mL}) \mathrm{un}-$ der a nitrogen atmosphere. The reaction mixture was stirred at $100{ }^{\circ} \mathrm{C}$ for 2 hours, and then cooled to room temperature. After removal of solvent under reduced pressure, the resulting precipitates were separated by filtration, washed with hexane $(3 \mathrm{~mL})$, and then dried under reduced pressure to give $2.01 \mathrm{~g}(1.60 \mathrm{mmol}, 94 \%)$ of 8 as a yellow ocher solid.

${ }^{1} \mathrm{H}$ NMR $\left(400 \mathrm{MHz}, \mathrm{CD}_{2} \mathrm{Cl}_{2}\right): \delta 2.70-2.86(\mathrm{~m}, 2 \mathrm{H}), 2.99(\mathrm{~s}, 3 \mathrm{H}), 3.11(\mathrm{~s}, 3 \mathrm{H}), 3.12-3.28(\mathrm{~m}, 2 \mathrm{H}), 3.30-3.48(\mathrm{~m}$, $4 \mathrm{H}), 4.27-4.45(\mathrm{~m}, 1 \mathrm{H}), 6.82-6.90(\mathrm{~m}, 2 \mathrm{H}), 7.70-7.80(\mathrm{~m}, 4 \mathrm{H}), 7.82-7.88(\mathrm{~m}, 4 \mathrm{H}), 7.90-7.98(\mathrm{~m}, 4 \mathrm{H}) .{ }^{31} \mathrm{P}$ NMR (161 MHz, $\left.\mathrm{CD}_{2} \mathrm{Cl}_{2}\right): \delta 48.9 .{ }^{13} \mathrm{C}$ NMR (125 MHz, $\mathrm{CD}_{2} \mathrm{Cl}_{2}$, selected peaks): $\delta 21,43(\mathrm{~s}), 22.40(\mathrm{~s}), 34,40(\mathrm{t}, J=$ $11.3 \mathrm{~Hz}$ ), 39,23 (s), 39.86 (s), 42.33 (s), 49.96 (m), 50.98 (s), 128.46 (s), 129.27 (s), 130.81 (m), 133.64 (m), 133.79 (s), $138.25(\mathrm{t}, \mathrm{J}=16.3 \mathrm{~Hz}), 139.24(\mathrm{t}, J=17.5 \mathrm{~Hz}), 177.65(\mathrm{t}, J=11.9 \mathrm{~Hz})$. HRMS (APCI, $m / z)$ calcd for $\mathrm{C}_{41} \mathrm{H}_{29} \mathrm{~N}_{3} \mathrm{~F}_{24} \mathrm{P}_{2} \mathrm{Cl}_{2} \mathrm{Ru}\left([\mathrm{M}]^{+}\right): 1252.9874$. Found: 1252.9870 . 


\section{Synthesis of Ruthenium Complex 9.}
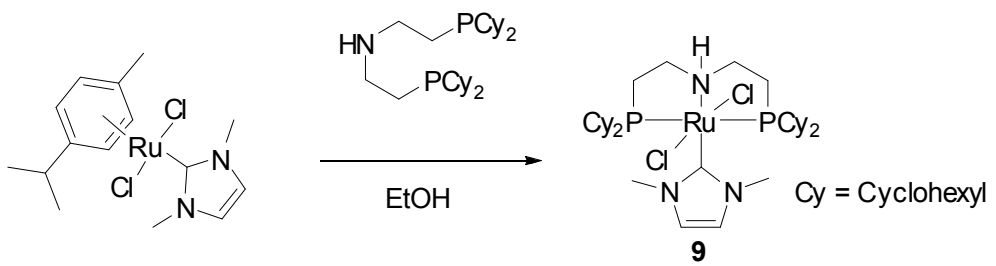

To a $20 \mathrm{~mL}$ Schlenk flask were added $\mathrm{RuCl}_{2}(p$-cymene $)(\mathrm{NHC}) \quad(80.5 \mathrm{mg}, \quad 0.20 \quad \mathrm{mmol})$, bis[2(dicyclohexylphosphanyl)ethyl]amine $(93.1 \mathrm{mg}, 0.20 \mathrm{mmol})$, and ethanol $(2.0 \mathrm{~mL})$ under a nitrogen atmosphere. The reaction mixture was stirred at $40{ }^{\circ} \mathrm{C}$ for 2 hours, and then cooled to room temperature. After removal of ethanol under reduced pressure, the precipitated solids were separated by filtration, washed with hexane $(3 \mathrm{~mL})$, and then dried under reduced pressure to give $41.0 \mathrm{mg}(0.056 \mathrm{mmol}, 28 \%)$ of 9 as a pink solid.

${ }^{1} \mathrm{H}$ NMR $\left(400 \mathrm{MHz}, \mathrm{CD}_{2} \mathrm{Cl}_{2}\right): \delta 1.00-3.10(\mathrm{~m}, 52 \mathrm{H}), 3.53(\mathrm{~s}, 3 \mathrm{H}), 3.67(\mathrm{~s}, 3 \mathrm{H}), 3.90-4.20(\mathrm{bs}, 1 \mathrm{H}), 6.70-6.80$ (m, $2 \mathrm{H}) .{ }^{31} \mathrm{P}$ NMR $\left(161 \mathrm{MHz}, \mathrm{CD}_{2} \mathrm{Cl}_{2}\right): \delta 43.1 .{ }^{13} \mathrm{C} \mathrm{NMR}\left(125 \mathrm{MHz}, \mathrm{CD}_{2} \mathrm{Cl}_{2}\right): \delta 25.71(\mathrm{t}, J=8.1 \mathrm{~Hz}), 27.11(\mathrm{~s}), 28.11$ (s), 28.47 (m), 29.03 (s), 29.92 (s), 30.47 (s), 30.59 (s), 32.14 (t, $J=6.9 \mathrm{~Hz}), 35.85$ (t, J=7.5 Hz), 41.41 (s), 42.01 (s), 48.51 (s), 122.81 (s), 122.87 (s), 183.26 (m). HRMS (ESI, m/z) calcd. for $\mathrm{C}_{33} \mathrm{H}_{61} \mathrm{~N}_{3} \mathrm{P}_{2} \mathrm{ClRu}\left([\mathrm{M}-\mathrm{Cl}]^{+}\right): 698.3073$. Found: 698.3047. 


\section{Hydrogenation of Esters \\ Procedures for Hydrogenation of Methyl Benzoate under a Hydrogen Pressure of 1.0 MPa.}

Complex $1(6.1 \mathrm{mg} 0.005 \mathrm{mmol})$ and a NHC precursor $(0.01 \mathrm{mmol})$ were added to a $100 \mathrm{~mL}$ autoclave with a magnetic stirring bar. After replacing atmosphere with nitrogen gas, $10 \mathrm{~mL}$ of toluene and methyl benzoate (136.15 mg, 10 $\mathrm{mmol})$ and a $1 \mathrm{M}$ THF solution of $\mathrm{KO}^{t} \mathrm{Bu}(1.0 \mathrm{~mL})$ were added. The autoclave was sealed, flushed with $\mathrm{H}_{2}$ four times, and placed under a hydrogen pressure of $1.0 \mathrm{MPa}$. After stirring at $80{ }^{\circ} \mathrm{C}$ for $5 \mathrm{~h}$, the reaction mixture was cooled to room temperature and hydrogen gas was carefully vented. The reaction mixture was analyzed by GC using decane or tridecane as an internal standard.

\section{Representative Procedures for Atmospheric Hydrogenation of Esters.}

\section{$<$ Hydrogenation of Methyl Benzoate $>$}

$\mathrm{RuCl}_{2}(\mathrm{NHC})(\mathrm{PNP})(6,14.2 \mathrm{mg}, 0.02 \mathrm{mmol})$ was added to a $40 \mathrm{~mL}$ Schlenk flask with a magnetic stirring bar. After replacing atmosphere with nitrogen gas, $2 \mathrm{~mL}$ of THF and methyl benzoate $(141.4 \mathrm{mg}, 1.04 \mathrm{mmol})$ and a $1 \mathrm{M}$ THF solution of $\mathrm{KO}^{t} \mathrm{Bu}(0.2 \mathrm{~mL})$ were added. The Schlenk flask was equipped with an adapter attached with a $\mathrm{H}_{2}$ balloon, and flushed with $\mathrm{H}_{2}$ four times. After stirring at $50{ }^{\circ} \mathrm{C}$ for $5 \mathrm{~h}$, the reaction mixture was cooled to room temperature and hydrogen gas was released. The reaction mixture was purified by column chromatography on silica gel (eluent: diethyl ether $/ n$-hexane $=1 / 4)$ to afford benzyl alcohol $(100.6 \mathrm{mg}, 0.93 \mathrm{mmol}, 90 \%)$ as a colorless oil. ${ }^{1} \mathrm{H} \mathrm{NMR}(400 \mathrm{MHz}$, $\left.\mathrm{CD}_{2} \mathrm{Cl}_{2}\right): \delta 1.80-2.00(\mathrm{bs}, 1 \mathrm{H}), 4.65(\mathrm{~s}, 3 \mathrm{H}), 7.22-7.40(\mathrm{~m}, 5 \mathrm{H}) .{ }^{13} \mathrm{C} \mathrm{NMR}\left(125 \mathrm{MHz}, \mathrm{CD}_{2} \mathrm{Cl}_{2}\right): \delta 65.06(\mathrm{~s}), 127.19$ (s), 127.67 (s), 128.68 (s), 141.50 (s). These NMR spectra were consistent with that of commercial reagent (Aldrich).

\section{$<$ Hydrogenation of Methyl Picolinate>}

$\mathrm{RuCl}_{2}(\mathrm{NHC})(\mathrm{PNP})(6,14.2 \mathrm{mg}, 0.02 \mathrm{mmol})$ was added to a $40 \mathrm{~mL}$ Schlenk flask with a magnetic stirring bar. After replacing atmosphere with nitrogen gas, $2 \mathrm{~mL}$ of THF and methyl picolinate (140.0 $\mathrm{mg}, 1.02 \mathrm{mmol})$ and a $1 \mathrm{M}$ THF solution of $\mathrm{KO}^{t} \mathrm{Bu}(0.2 \mathrm{~mL})$ were added. The Schlenk flask was equipped with an adapter attached with a $\mathrm{H}_{2}$ balloon, and flushed with $\mathrm{H}_{2}$ four times. After stirring at $50{ }^{\circ} \mathrm{C}$ for $5 \mathrm{~h}$, the reaction mixture was cooled to room temperature and hydrogen gas was released. The reaction mixture was purified by column chromatography on silica gel (eluent: diethyl ether) to afford 2-pyridinemethanol $(109.5 \mathrm{mg}, 1.00 \mathrm{mmol}, 93 \%)$ as a colorless oil. ${ }^{1} \mathrm{H}$ NMR $\left(400 \mathrm{MHz}^{\mathrm{C}} \mathrm{CD}_{2} \mathrm{Cl}_{2}\right): \delta$ $3.60-4.20(\mathrm{bs}, 1 \mathrm{H}), 4.71(\mathrm{~s}, 3 \mathrm{H}), 7.18-7.30(\mathrm{~m}, 2 \mathrm{H}), 7.70(\mathrm{dt}, J=1.9,7.7 \mathrm{~Hz}, 1 \mathrm{H}) .8 .50-8.56(\mathrm{~m}, 1 \mathrm{H}) .{ }^{13} \mathrm{C} \mathrm{NMR}$ (125 MHz, $\mathrm{CD}_{2} \mathrm{Cl}_{2}$ ): $\delta 64.57$ (s), 120.96 (s), 122.45 (s), 137.07 (s), 148.66 (s), 160.50 (s). These NMR spectra were consistent with that of commercial reagent (Wako).

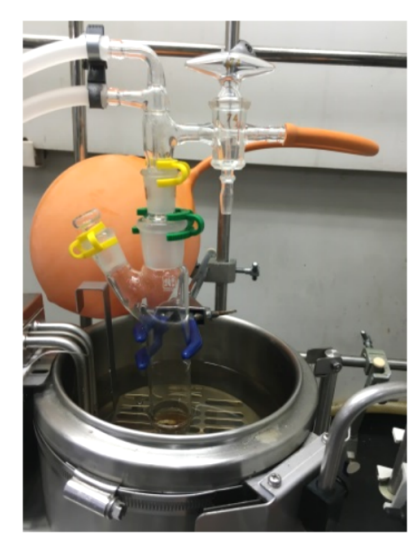

Figure S1. Experimental Setup for the Atmospheric Hydrogenation of Esters. 
Table S1. Effect of Temperature and Concentration in Atmospheric Hydrogenation of Esters.

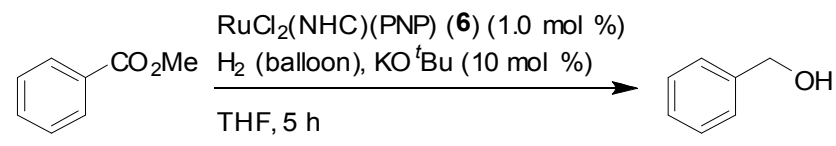

$\begin{array}{cccccc}\text { entry } & \text { temp, }{ }^{\circ} \mathrm{C} & \text { conc, } \mathrm{M} & \begin{array}{c}\% \text { conv }^{\mathrm{b}} \\ \% \text { yield }^{\mathrm{b}}\end{array} & \begin{array}{c}\% \text { yield }^{\mathrm{b}} \\ \mathrm{PhCH}_{2} \mathrm{OH}\end{array} & \mathrm{PhCO}_{2} \mathrm{CH}_{2} \mathrm{Ph} \\ 1 & 50 & 1.0 & 92 & 74 & 4 \\ 2 & 55 & 1.0 & 94 & 60 & 11 \\ 3 & 45 & 1.0 & 93 & 72 & 6 \\ 4 & 50 & 2.0 & 82 & 58 & 8 \\ 5 & 50 & 0.5 & 93 & 80 & 2\end{array}$

${ }^{\mathrm{a}}$ Reaction conditions; substrate $(1.0 \mathrm{mmol}), 6(0.01 \mathrm{mmol}), \mathrm{H}_{2}$ (balloon), $\mathrm{KO}{ }^{t} \mathrm{Bu}(0.1 \mathrm{mmol}), \mathrm{THF}, 50{ }^{\circ} \mathrm{C}$, for $5 \mathrm{~h}$.

${ }^{\mathrm{b}}$ Conversion and yield were determined by GC.

Table S2. Hydrogenation of Esters under 0.4 MPa of Hydrogen Pressure.

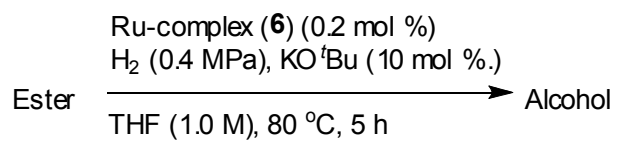

entry substrate

${ }^{\mathrm{a}}$ Reaction conditions; substrate $(1.0 \mathrm{mmol}), 6(0.002 \mathrm{mmol}), \mathrm{H}_{2}(0.4 \mathrm{MPa}), \mathrm{KO}^{t} \mathrm{Bu}(0.1 \mathrm{mmol}), \mathrm{THF}(1.0 \mathrm{~mL}), 80^{\circ} \mathrm{C}$, for $5 \mathrm{~h}$.

${ }^{\mathrm{b}}$ Conversion and yield were determined by GC. 


\section{X-ray Structure Determination for 7.}

Measurements were made on a Rigaku Saturn 724 diffractometer equipped with multiplayer mirror monochromated Mo- $K \alpha$ radiation $(\lambda=0.71070 \AA)$ under nitrogen stream at $123 \mathrm{~K}$. Indexing was performed from eighteen images. The crystal-to-detector distance was $45.02 \mathrm{~mm}$. The data were collected to a maximum $2 \theta$ value of $55.0^{\circ}$. A total of 720 oscillation images were collected. A sweep of data was carried out using $\omega$ scans from -110.0 to $70.0^{\circ}$ in $0.5^{\circ}$ steps, at $\chi=45.0^{\circ}$ and $\phi=0.0^{\circ}$. A second sweep was performed using $\omega$ scans from -110.0 to $70.0^{\circ}$ in $0.5^{\circ}$ steps, at $\chi$ $=45.0^{\circ}$ and $\phi=90.0^{\circ}$. Intensity data were collected for Lorentz-polarisation effects as well as absorption. Structure solution and refinements were performed with the CrystalStructure program package. The heavy atom positions were determined by direct methods (SIR2002), and the remaining non-hydrogen atoms were found by subsequent Fourier techniques. An empirical absorption correction based on equivalent reflections was applied to all data. All nonhydrogen atoms other than solvent molecules were refined anisotropically by full-matrix least-square techniques based on $F^{2}$. All hydrogen atoms were constrained to ride on their parent atom. Relevant crystallographic data are compiled in Table $\mathrm{S} 1$.

Table S3. Crystallographic Data for 7

\begin{tabular}{|c|c|}
\hline & 7 \\
\hline Empirical Formula & $\mathrm{C}_{35} \mathrm{H}_{40} \mathrm{Cl}_{2} \mathrm{~N}_{4} \mathrm{P}_{2} \mathrm{Ru}$ \\
\hline Formula Weight & 750.65 \\
\hline Crystal Color, Habit & yellow, prism \\
\hline Crystal System & Triclinic \\
\hline Space Group & $P-1(\# 2)$ \\
\hline \multirow[t]{7}{*}{ Lattice Parameters } & $a=9.834(2) \AA$ \\
\hline & $b=10.604(3) \AA$ \\
\hline & $c=16.861(4) \AA$ \\
\hline & $\alpha=85.889(7)^{\circ}$ \\
\hline & $\beta=78.666(6)^{\circ}$ \\
\hline & $\gamma=81.528(6)^{\circ}$ \\
\hline & $V=1703.5(7) \AA^{3}$ \\
\hline$Z$ value & 2 \\
\hline$D_{\text {calc }}$ & $1.463 \mathrm{~g} / \mathrm{cm}^{3}$ \\
\hline $\mathrm{F}_{000}$ & 772.00 \\
\hline$\mu(\mathrm{MoK} \alpha)$ & $7.421 \mathrm{~cm}^{-1}$ \\
\hline Exposure Rate & $16.0 \mathrm{sec} . / \mathrm{o}$ \\
\hline No. of Reflections Measured & 14059 \\
\hline No. of Unique Reflections & 7521 \\
\hline No. Variables & 437 \\
\hline$R 1(\mathrm{I}>2.00 \sigma(I))$ & 0.0369 \\
\hline $\mathrm{w} R 2$ (All reflections) & 0.0958 \\
\hline GOF on $F^{2}$ & 1.000 \\
\hline
\end{tabular}


${ }^{1} \mathrm{H},{ }^{31} \mathrm{P},{ }^{13} \mathrm{C}$ NMR, and HRMS Spectra

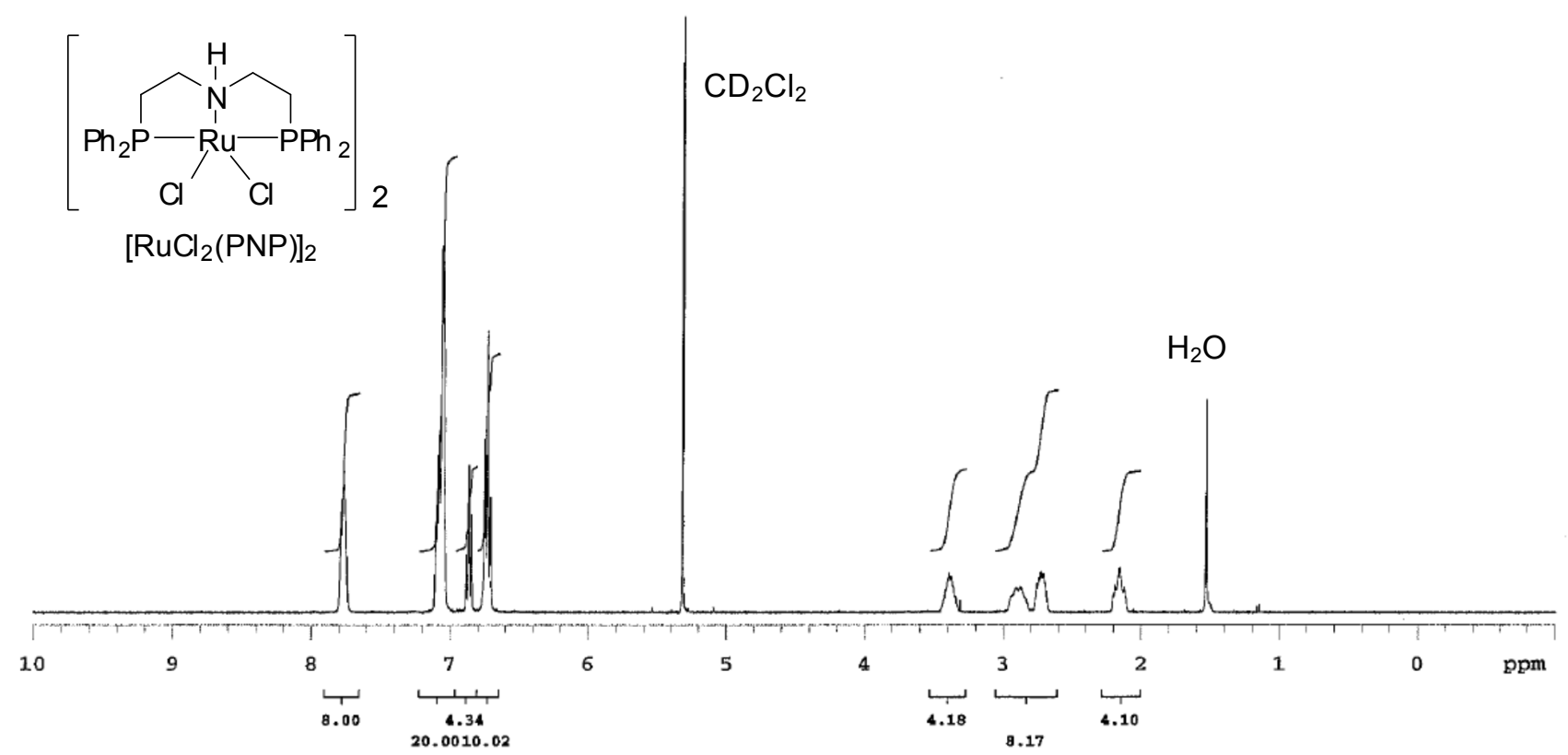

Figure S2. ${ }^{1} \mathrm{H}$ NMR Spectrum of $\left[\mathrm{RuCl}_{2}(\mathrm{PNP})\right]_{2}(\mathbf{1})$ in $\mathrm{CD}_{2} \mathrm{Cl}_{2}$.

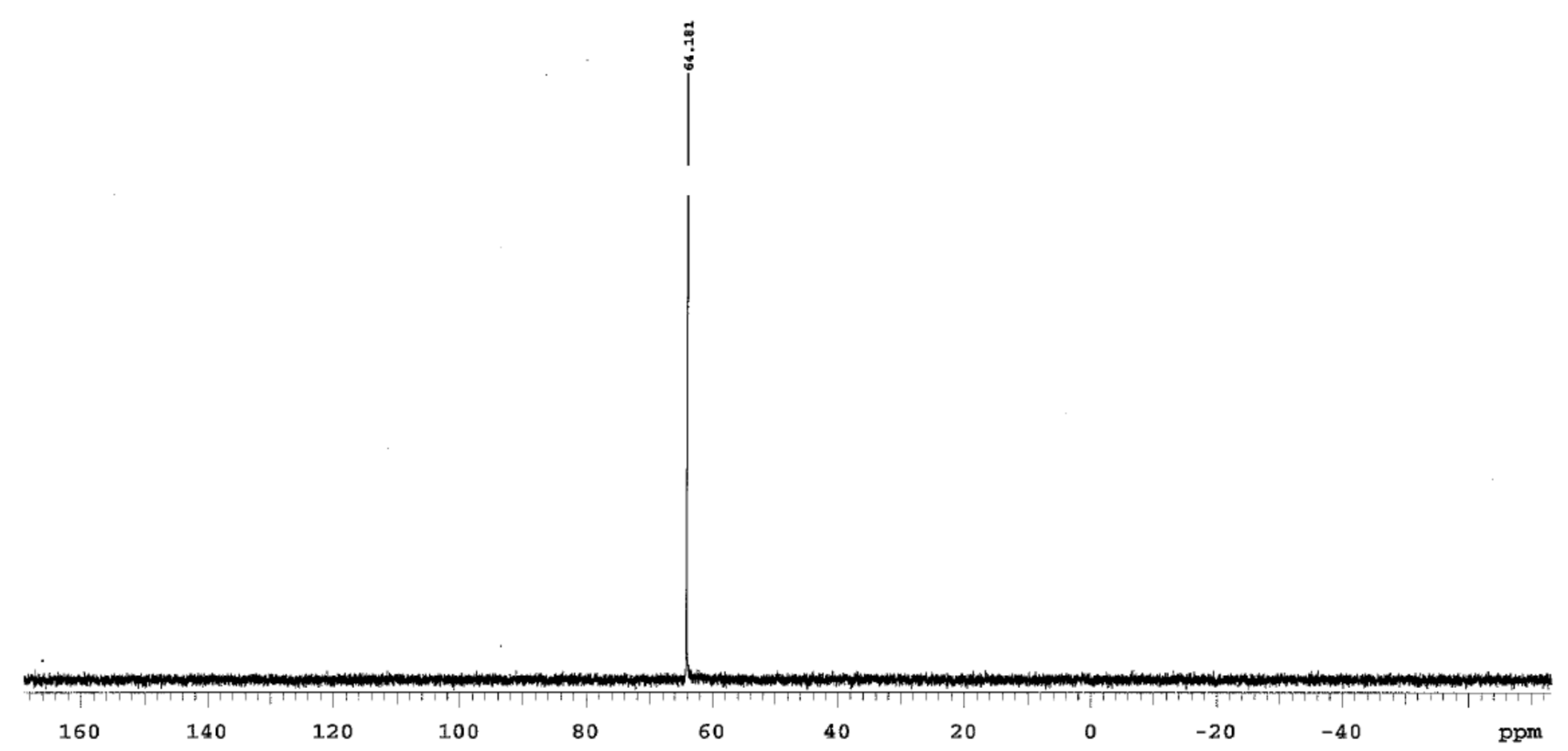

Figure S3. ${ }^{31} \mathrm{P}\left\{{ }^{1} \mathrm{H}\right\}$ NMR Spectrum of $\left[\mathrm{RuCl}_{2}(\mathrm{PNP})\right]_{2}(\mathbf{1})$ in $\mathrm{CD}_{2} \mathrm{Cl}_{2}$. 


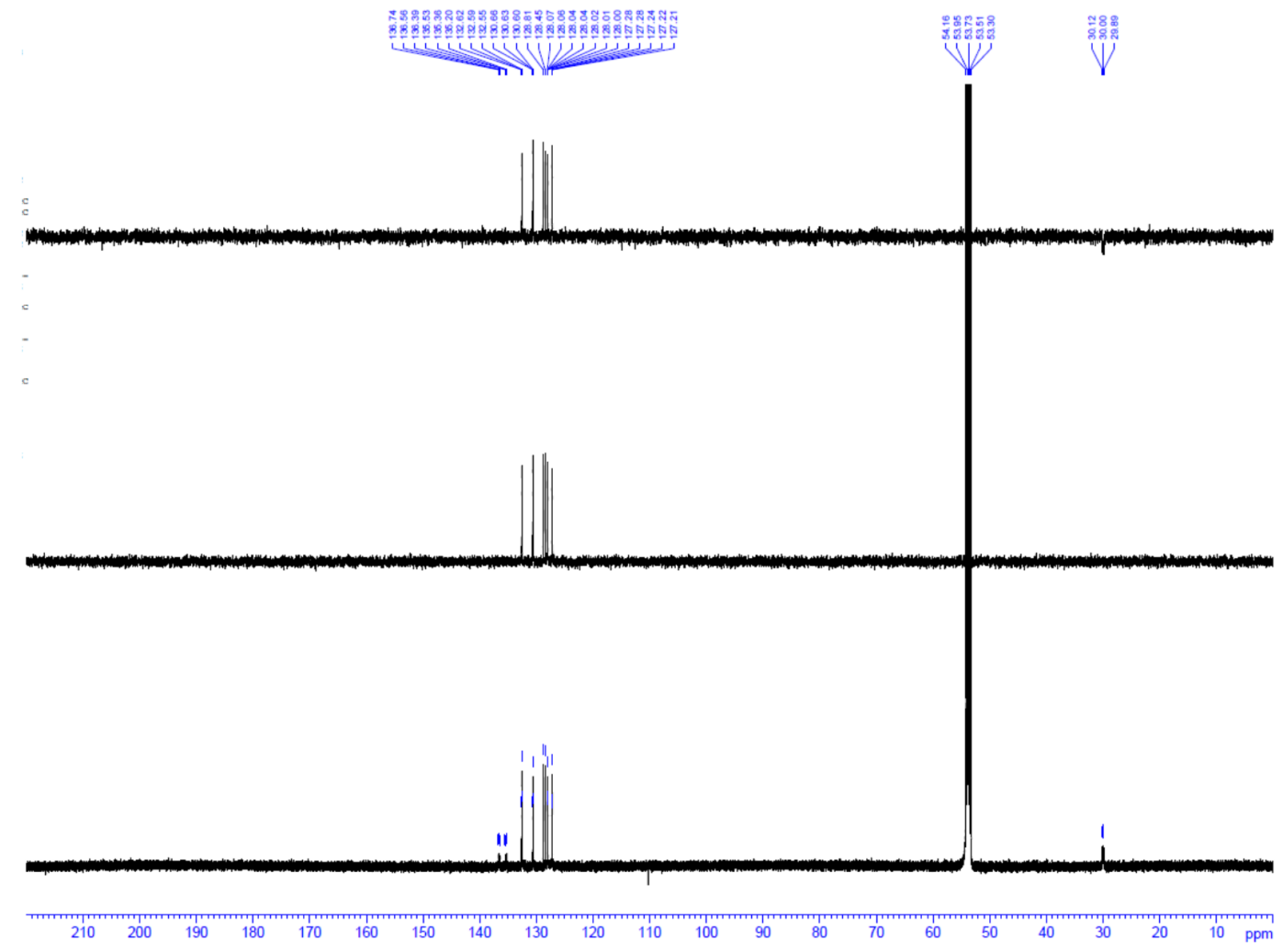

Figure S4. ${ }^{13} \mathrm{C}\left\{{ }^{1} \mathrm{H}\right\}$ NMR Spectrum of $\left[\mathrm{RuCl}_{2}(\mathrm{PNP})\right]_{2}(\mathbf{1})$ in $\mathrm{CD}_{2} \mathrm{Cl}_{2}$.
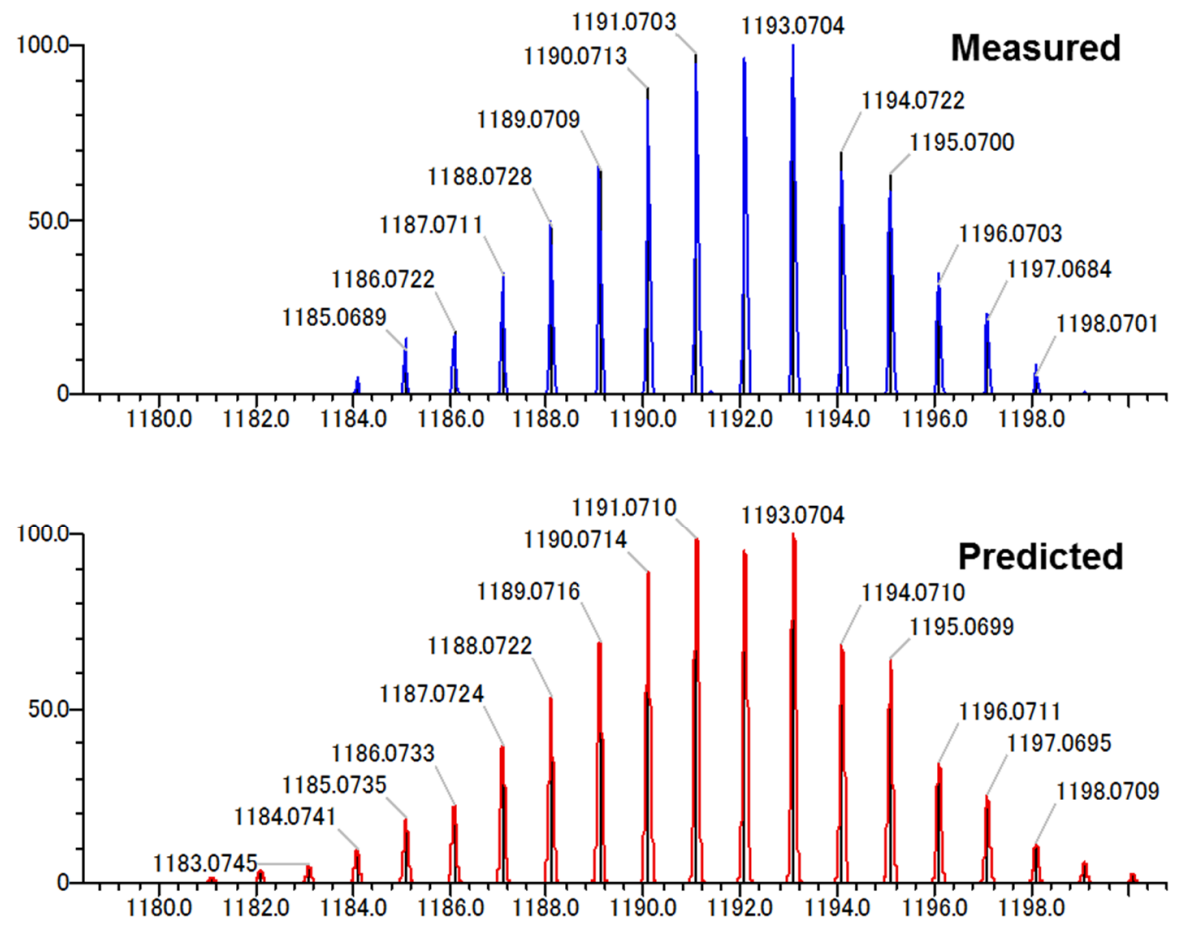

found $\mathrm{m} / \mathrm{z} 1191.0703[\mathrm{M}-\mathrm{Cl}]^{+}$

calcd. for $\left[\mathrm{C}_{56} \mathrm{H}_{58} \mathrm{~N}_{2} \mathrm{P}_{4} \mathrm{Cl}_{3} \mathrm{Ru}_{2}\right]^{+}(1191.0710,-0.7 \mathrm{mDa},-0.59 \mathrm{ppm})$

Figure S5. HRMS Spectrum of $\left[\mathrm{RuCl}_{2}(\mathrm{PNP})\right]_{2}(\mathbf{1})$. 

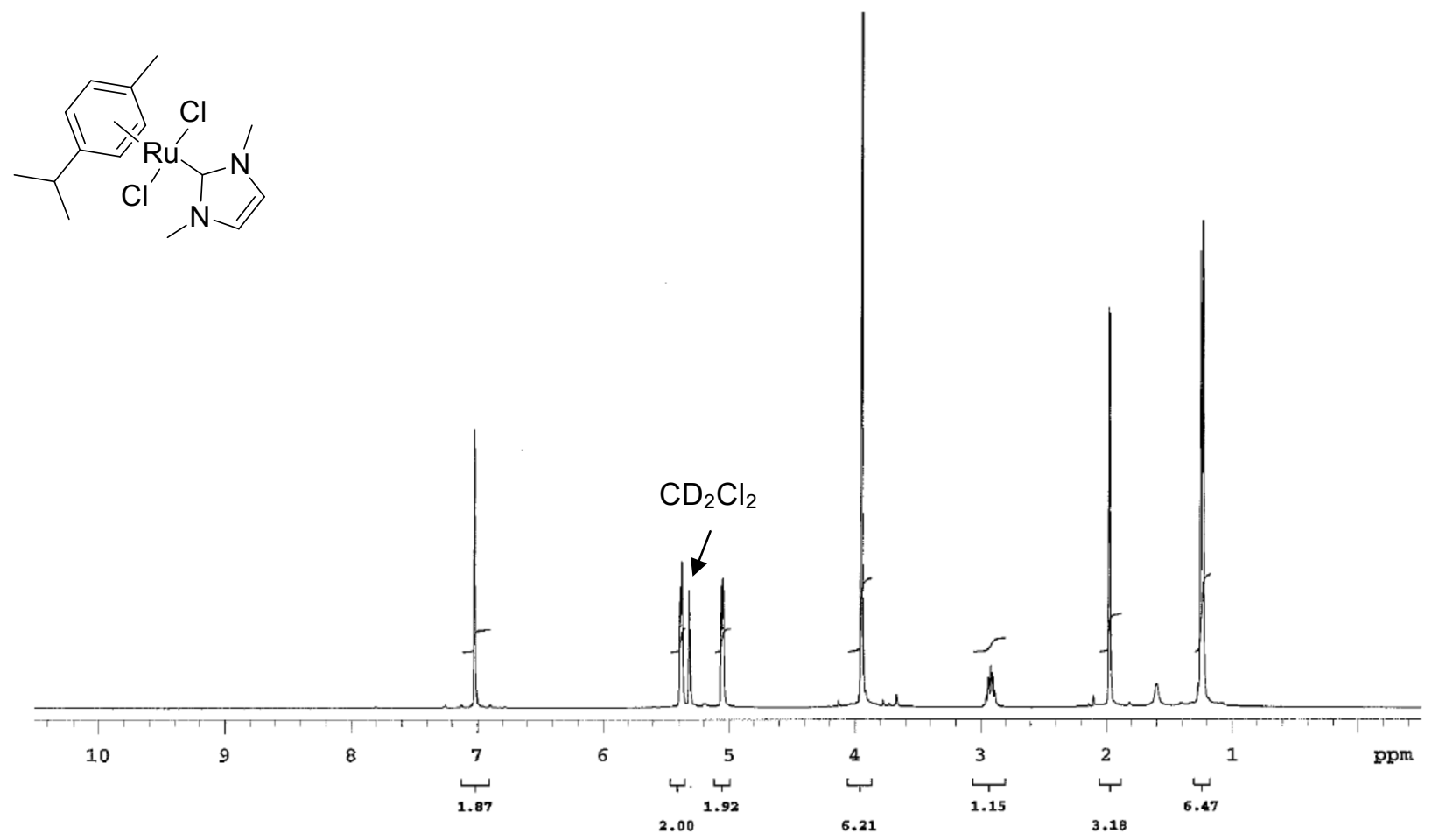

Figure S6. ${ }^{1} \mathrm{H}$ NMR Spectrum of $\mathrm{RuCl}_{2}\left(p\right.$-cymene)(NHC) in $\mathrm{CD}_{2} \mathrm{Cl}_{2}$.

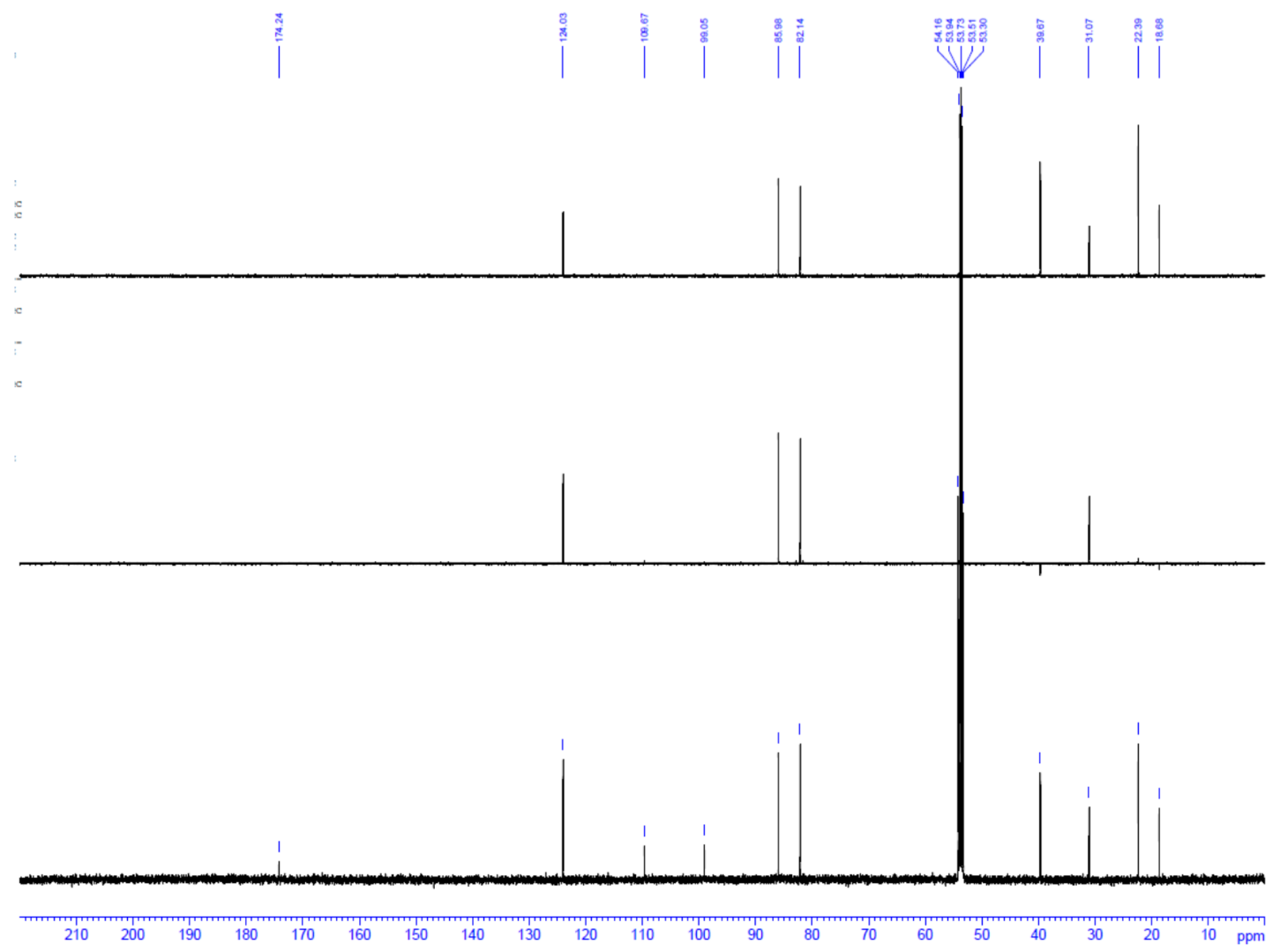

Figure S7. ${ }^{13} \mathrm{C}\left\{{ }^{1} \mathrm{H}\right\}$ NMR Spectrum of $\mathrm{RuCl}_{2}\left(p\right.$-cymene)(NHC) in $\mathrm{CD}_{2} \mathrm{Cl}_{2}$. 

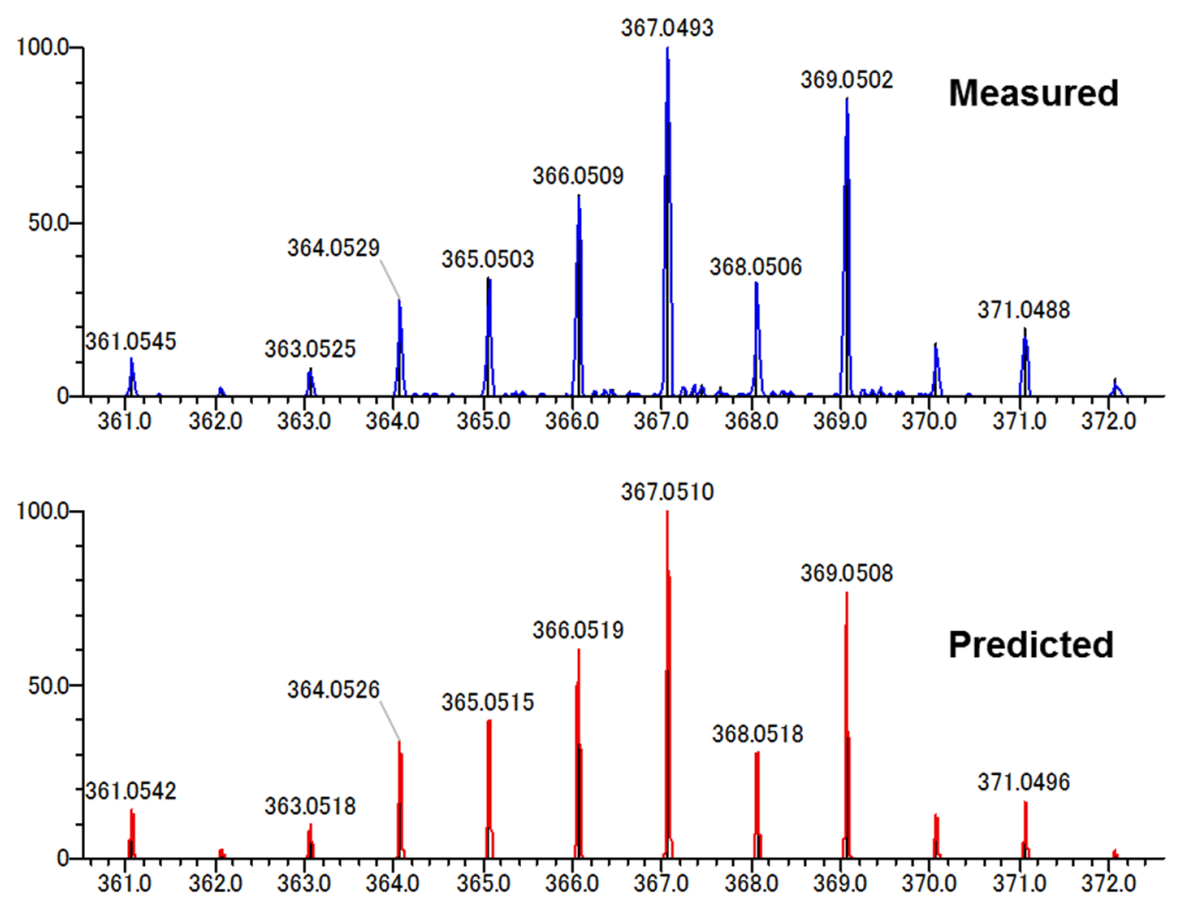

found $\mathrm{m} / \mathrm{z} 367.0493$ [M]

calcd. for $\left[\mathrm{C}_{15} \mathrm{H}_{22} \mathrm{~N}_{2} \mathrm{ClRu}\right]^{+}(367.0510,-1.7 \mathrm{mDa},-4.63 \mathrm{ppm})$

Figure S8. HRMS Spectrum of $\mathrm{RuCl}_{2}(p$-cymene)(NHC). 


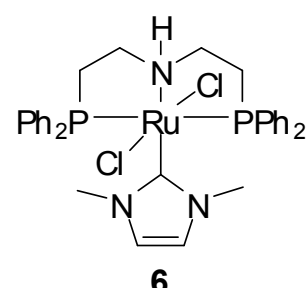

6

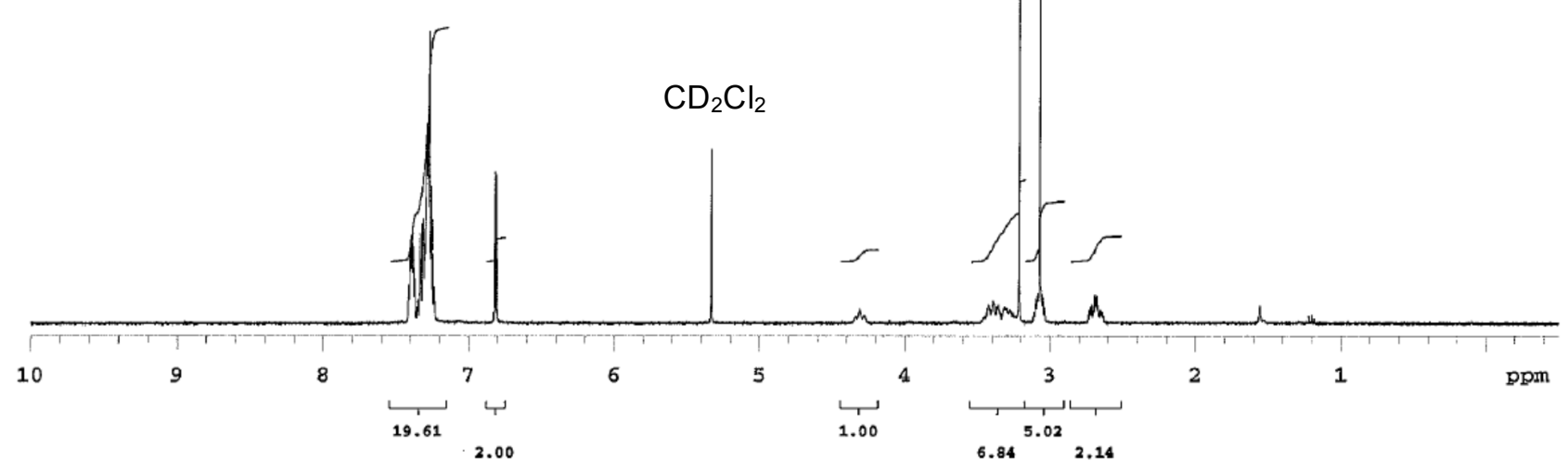

Figure S9. ${ }^{1} \mathrm{H}$ NMR Spectrum of Ruthenium Complex 6 in $\mathrm{CD}_{2} \mathrm{Cl}_{2}$.

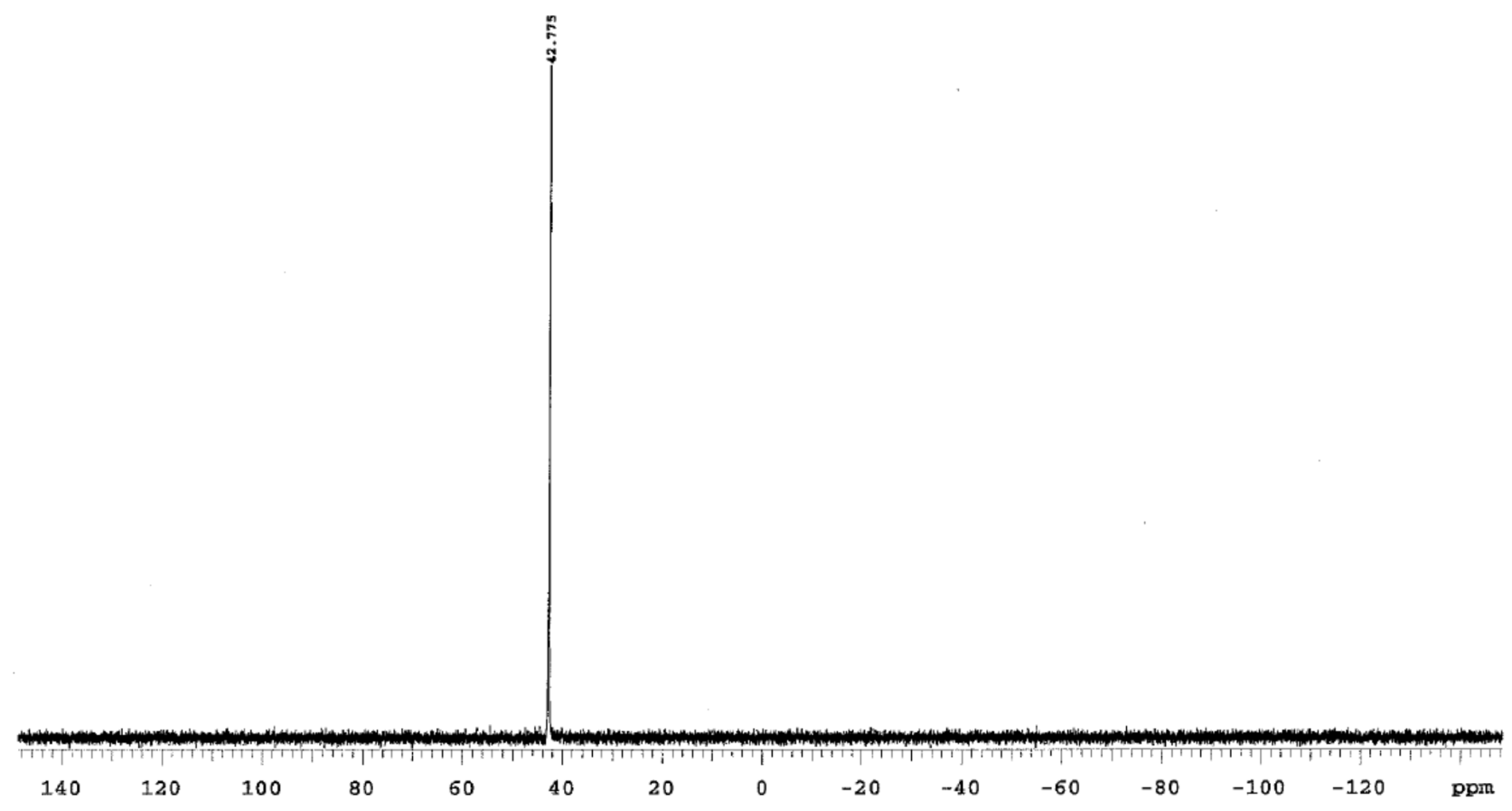

Figure S10. ${ }^{31} \mathrm{P}\left\{{ }^{1} \mathrm{H}\right\}$ NMR Spectrum of Ruthenium Complex 6 in $\mathrm{CD}_{2} \mathrm{Cl}_{2}$. 


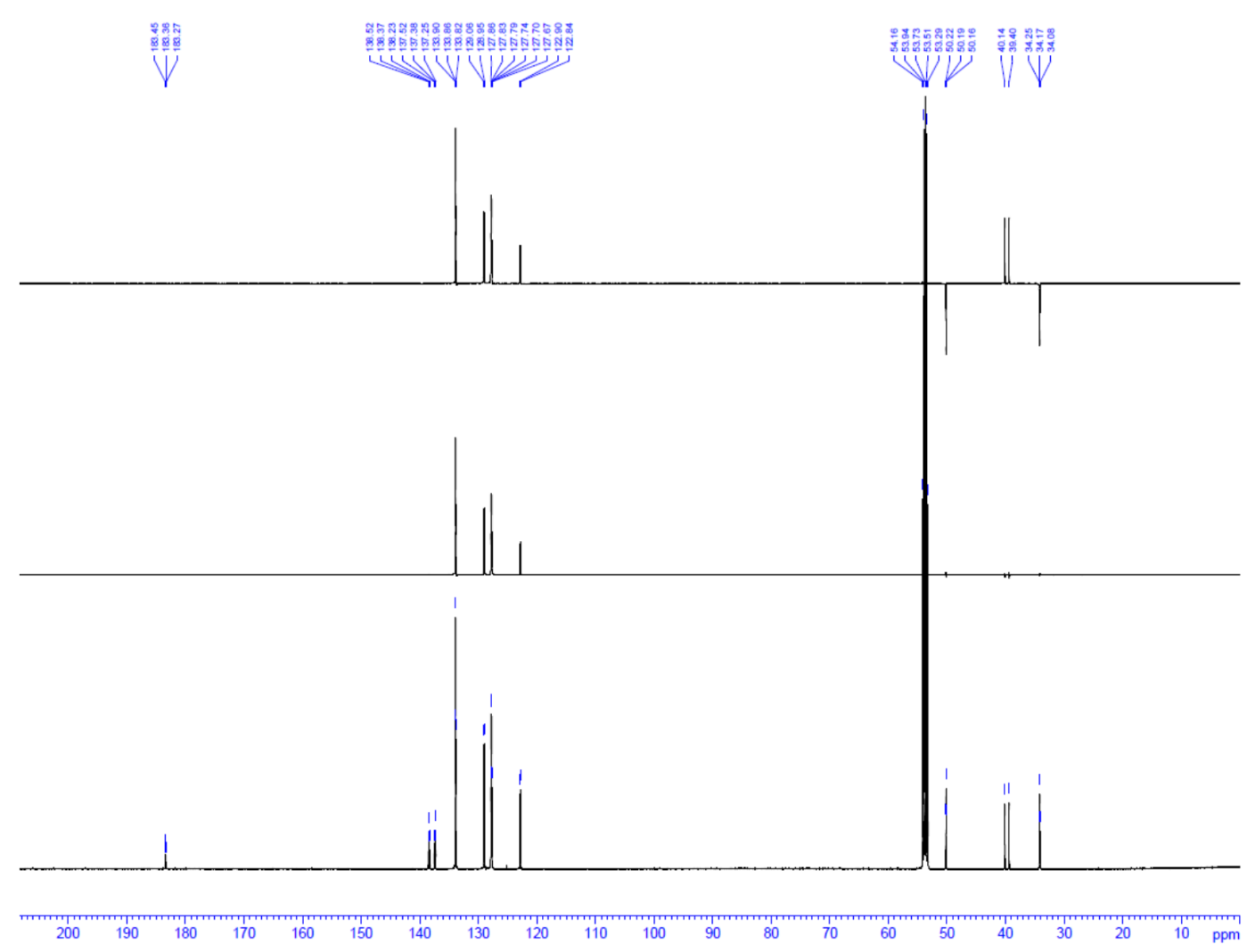

Figure S11. ${ }^{13} \mathrm{C}\left\{{ }^{1} \mathrm{H}\right\}$ NMR Spectrum of Ruthenium Complex 6 in $\mathrm{CD}_{2} \mathrm{Cl}_{2}$.
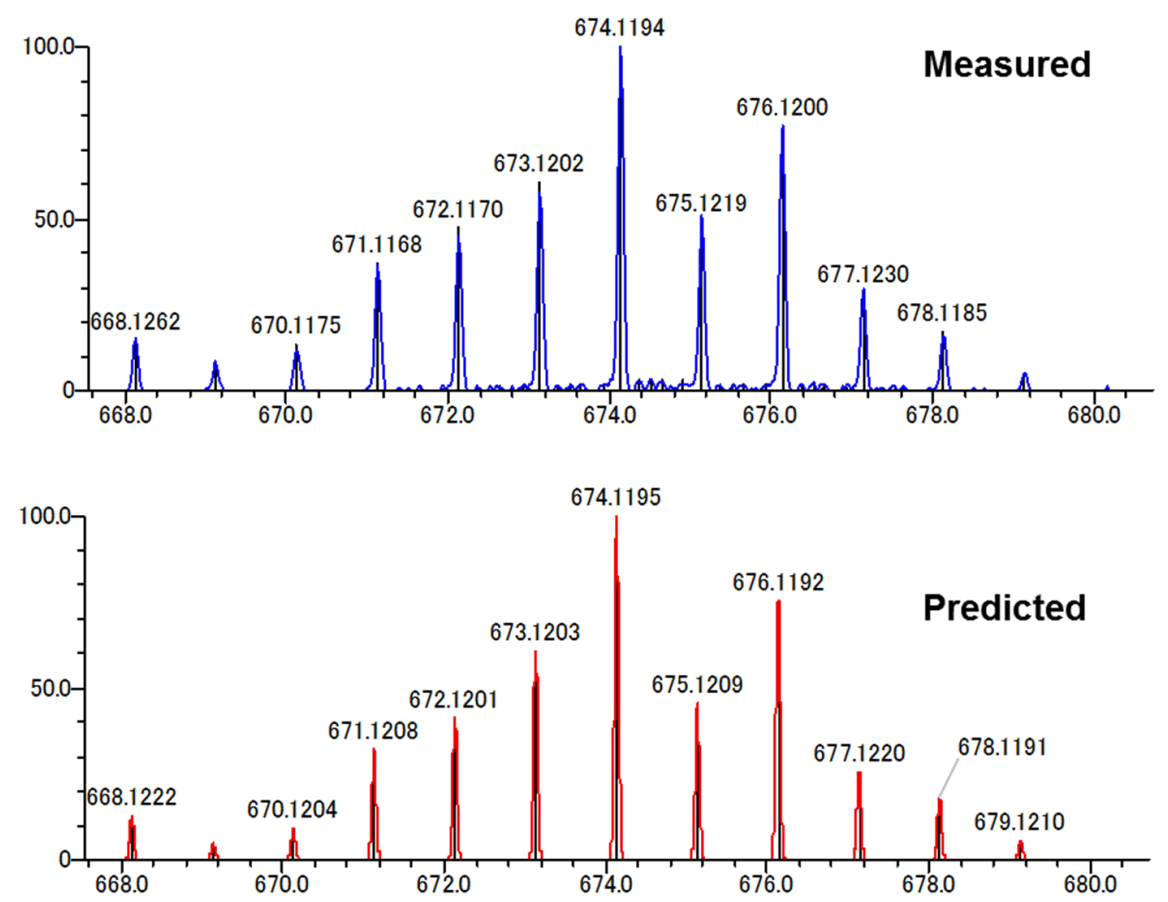

found $\mathrm{m} / \mathrm{z} 674.1194[\mathrm{M}]^{+}$

calcd. for $\left[\mathrm{C}_{33} \mathrm{H}_{37} \mathrm{~N}_{3} \mathrm{P}_{2} \mathrm{CIRu}\right]^{+}$(674.1195, -0.1 mDa, $\left.-0.15 \mathrm{ppm}\right)$

Figure S12. HRMS Spectrum of Ruthenium Complex 6. 


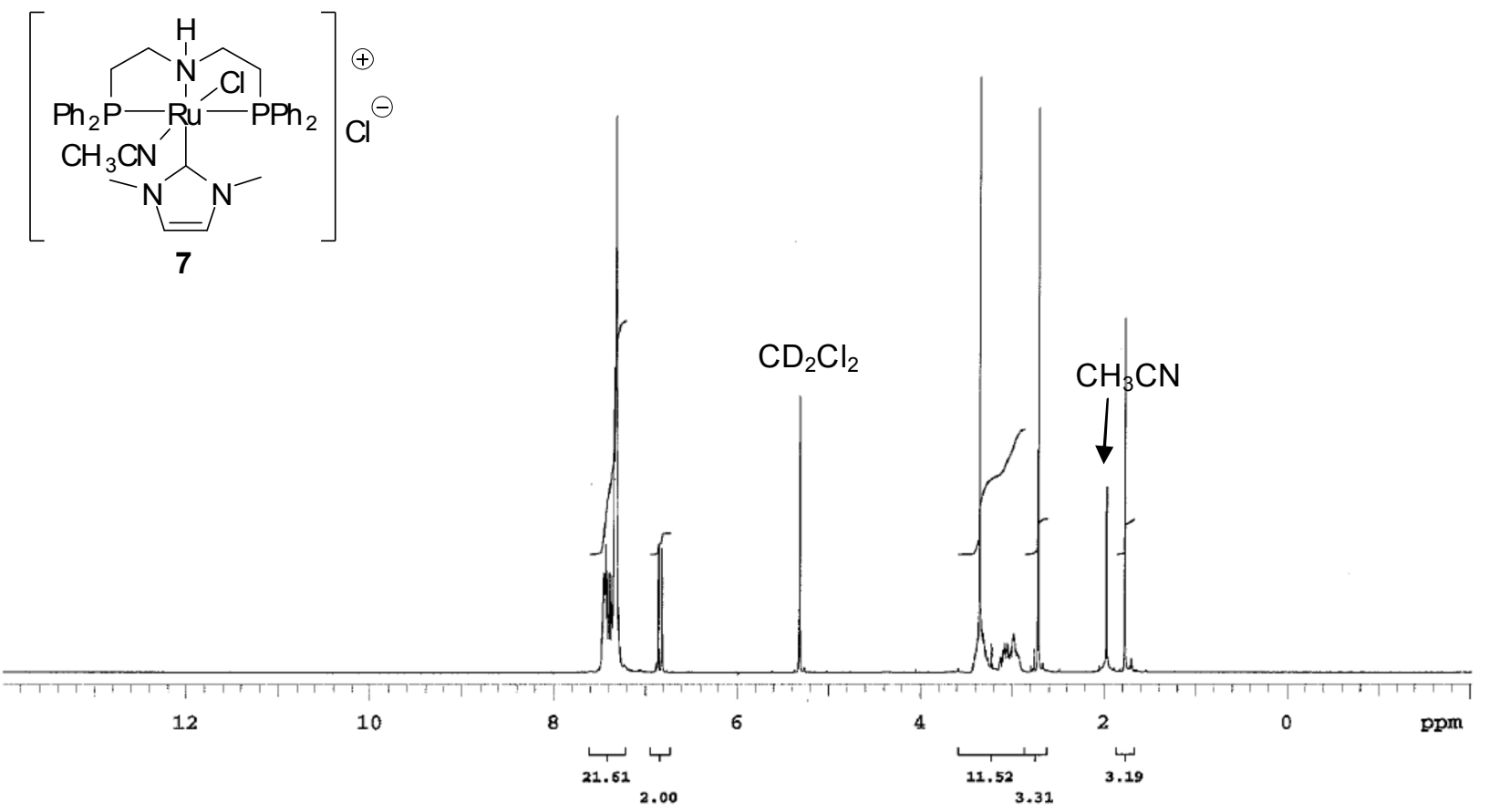

Figure S13. ${ }^{1} \mathrm{H}$ NMR Spectrum of Ruthenium Complex 7 in $\mathrm{CD}_{2} \mathrm{Cl}_{2}$.

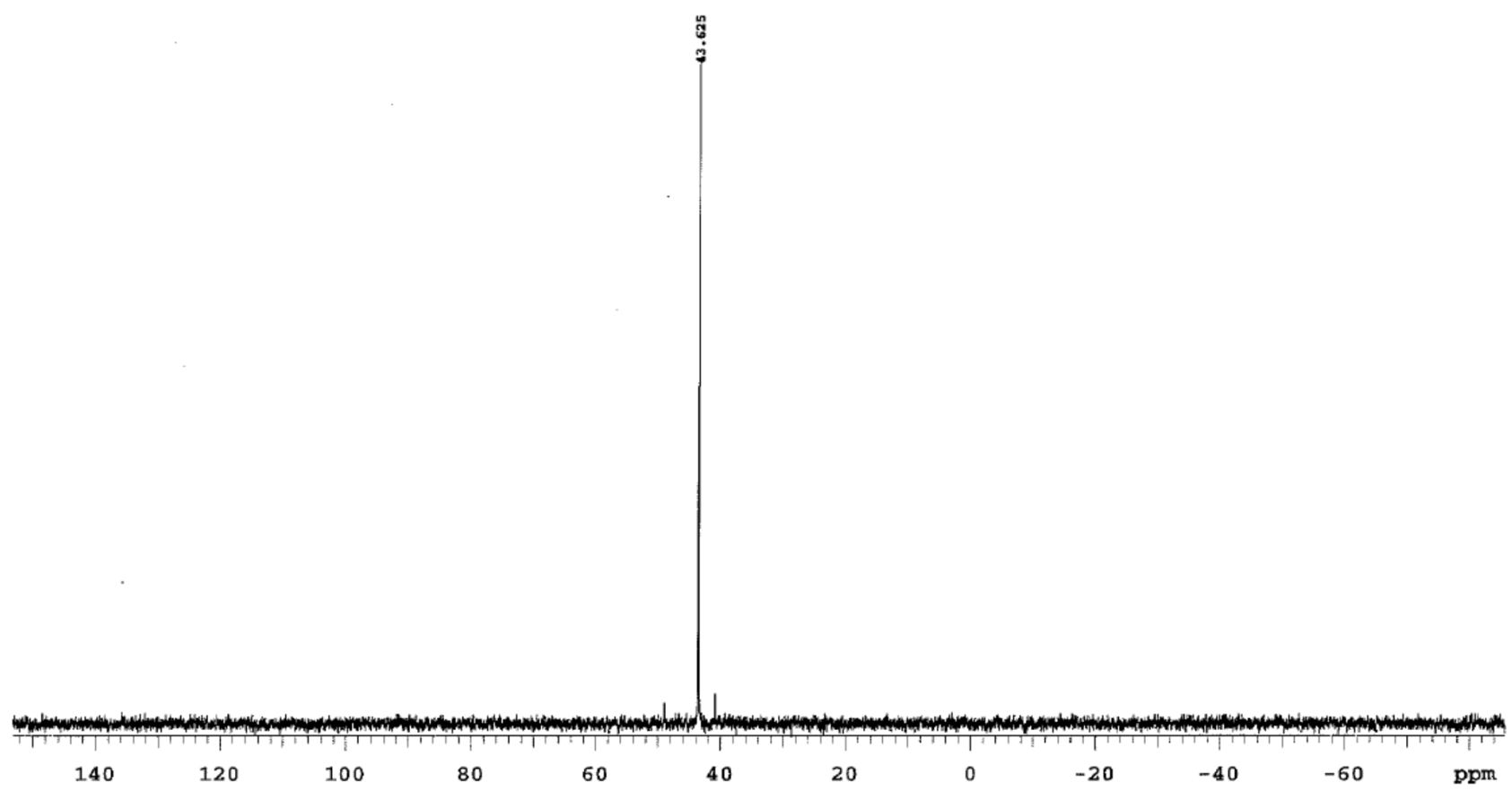

Figure S14. ${ }^{31} \mathrm{P}\left\{{ }^{1} \mathrm{H}\right\}$ NMR Spectrum of Ruthenium Complex 7 in $\mathrm{CD}_{2} \mathrm{Cl}_{2}$. 


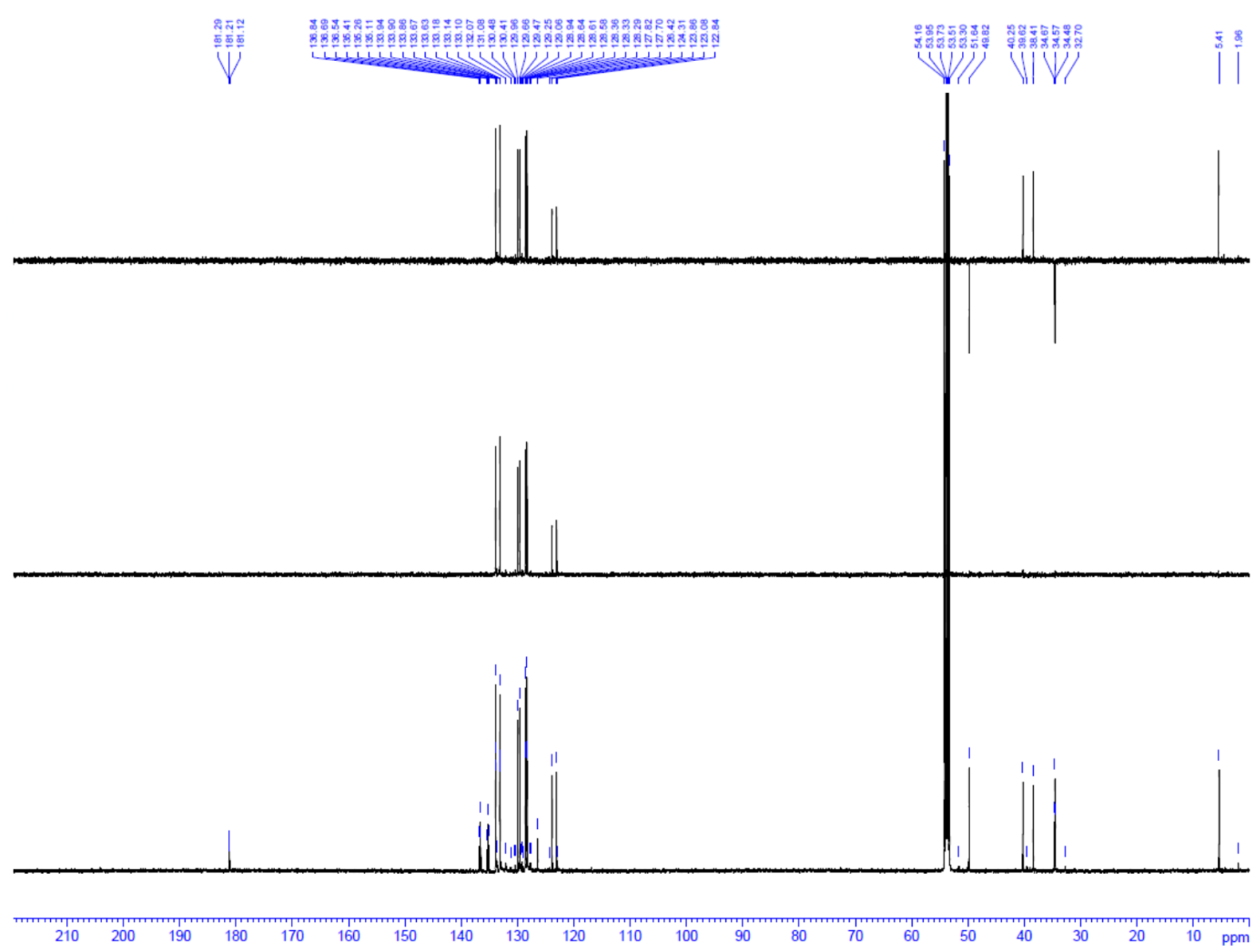

Figure S15. ${ }^{13} \mathrm{C}\left\{{ }^{1} \mathrm{H}\right\}$ NMR Spectrum of Ruthenium Complex 7 in $\mathrm{CD}_{2} \mathrm{Cl}_{2}$.

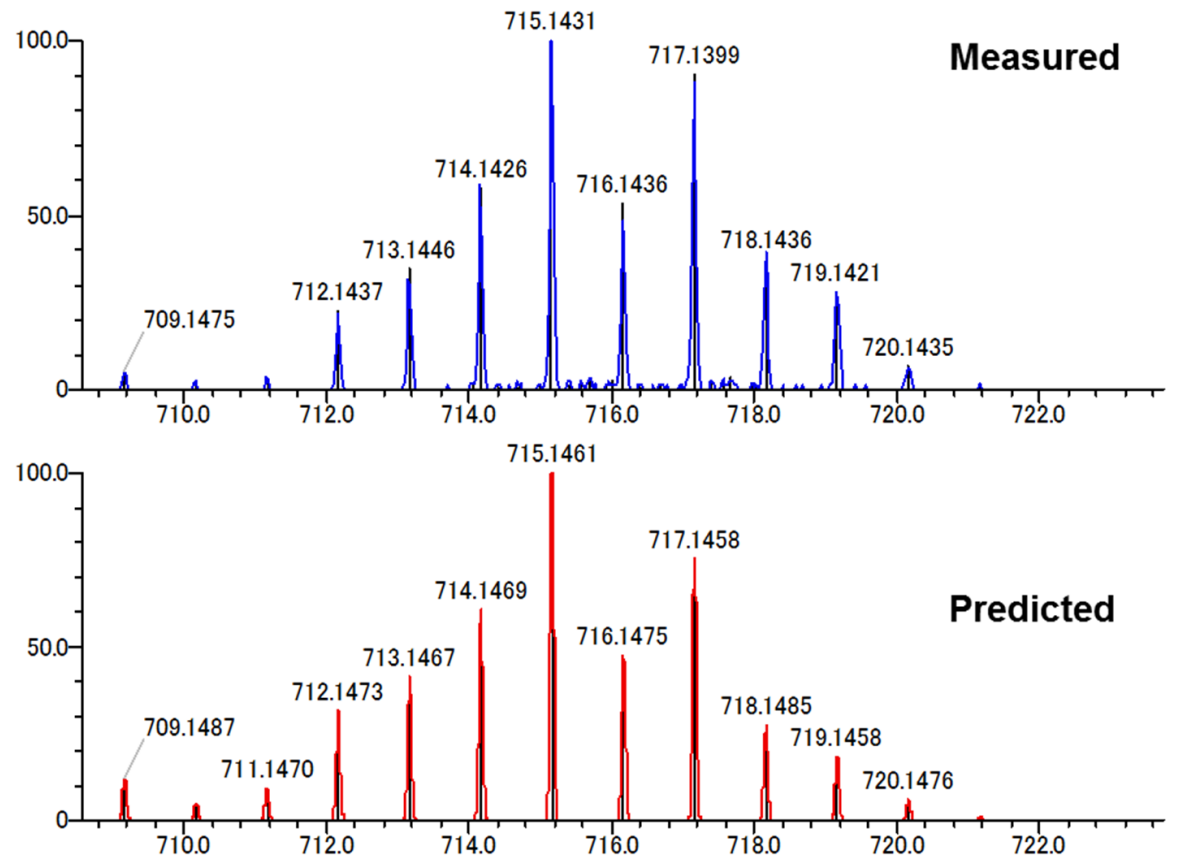

found $\mathrm{m} / \mathrm{z} 715.1431$ [M-Cl] ${ }^{+}$

calcd. for $\left[\mathrm{C}_{35} \mathrm{H}_{40} \mathrm{~N}_{4} \mathrm{P}_{2} \mathrm{CIRu}\right]^{+}$(715.1461, -3.0 mDa, -4.19 ppm)

Figure S16. HRMS Spectrum of Ruthenium Complex 7. 


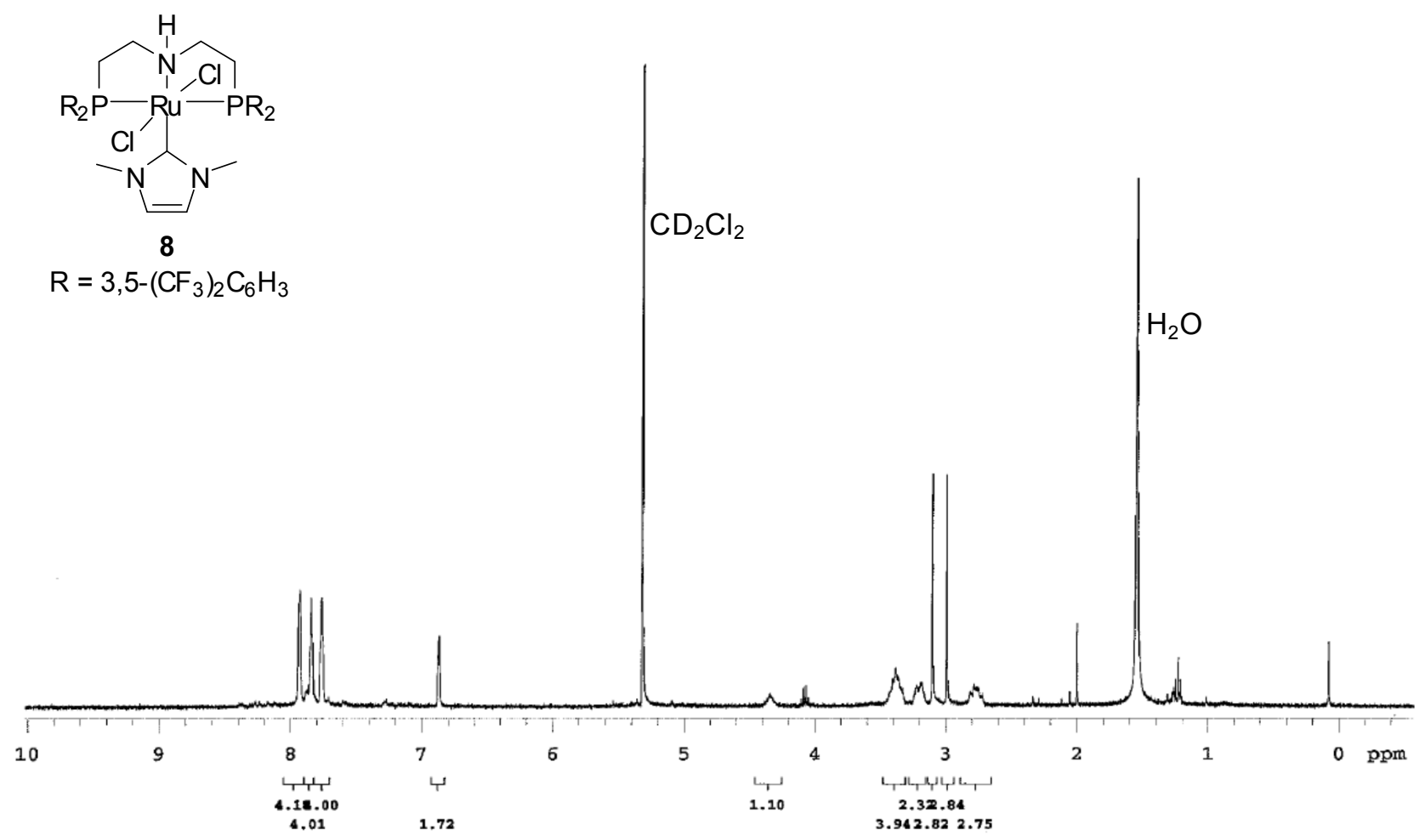

Figure S17. ${ }^{1} \mathrm{H}$ NMR Spectrum of Ruthenium Complex 8 in $\mathrm{CD}_{2} \mathrm{Cl}_{2}$.

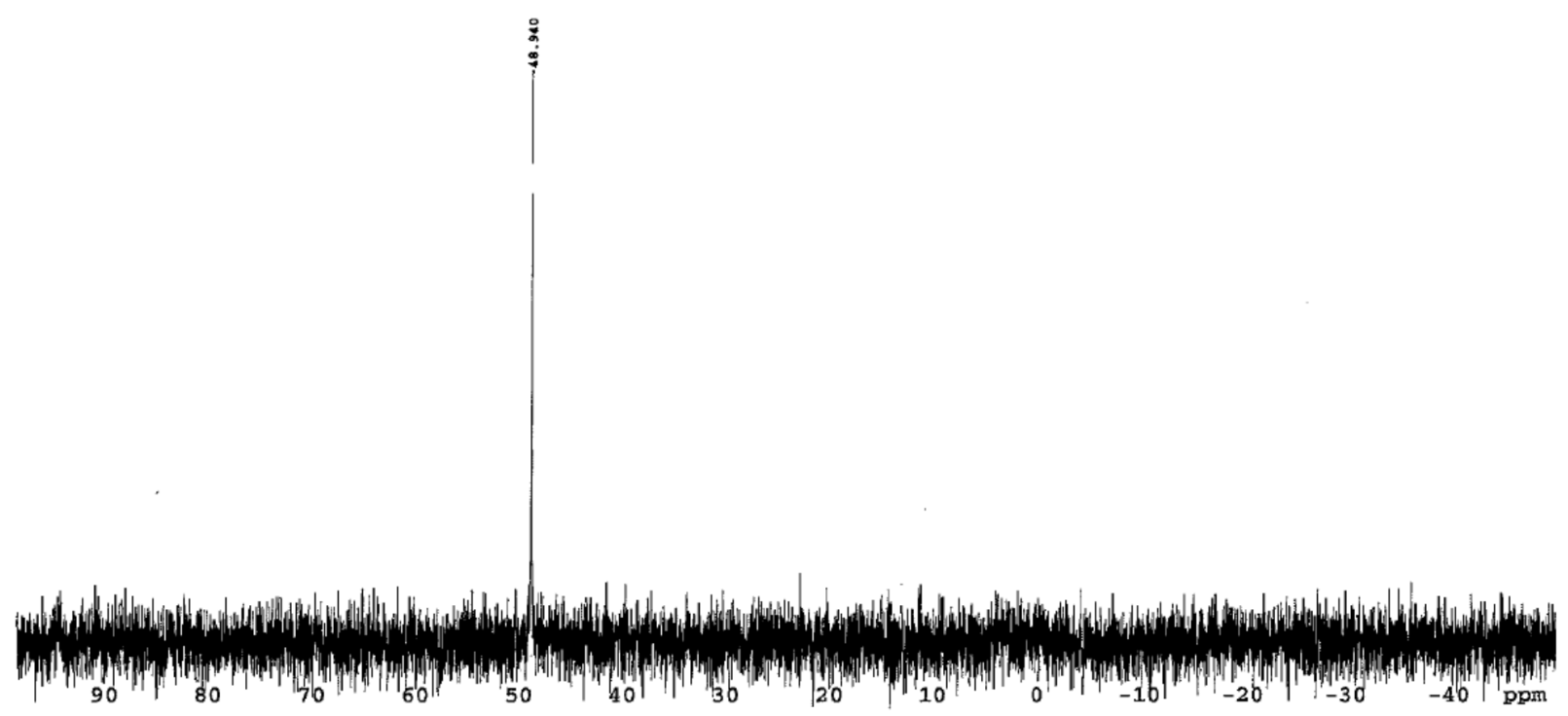

Figure S18. ${ }^{31} \mathrm{P}\left\{{ }^{1} \mathrm{H}\right\}$ NMR Spectrum of Ruthenium Complex 8 in $\mathrm{CD}_{2} \mathrm{Cl}_{2}$. 


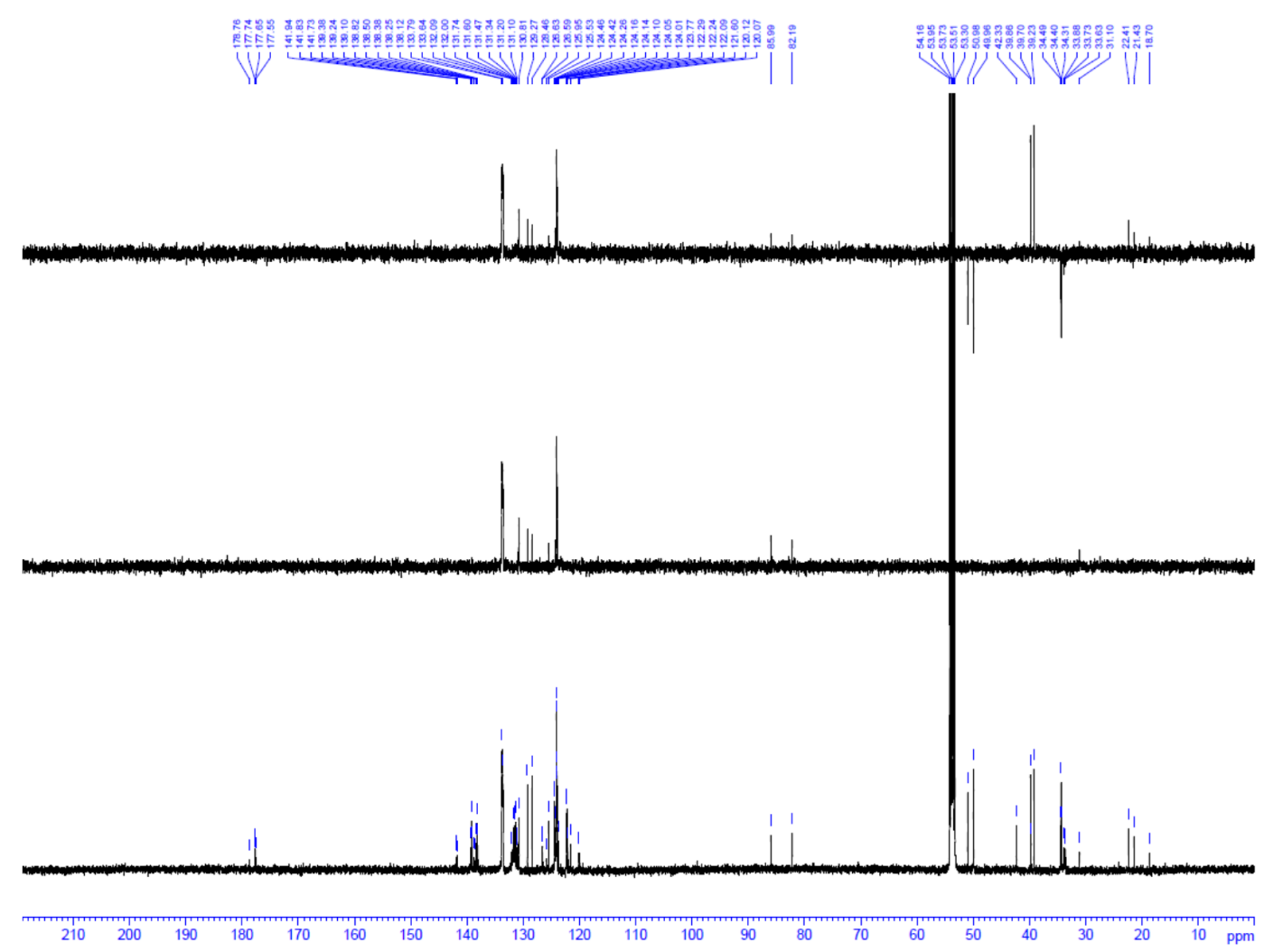

Figure S19. ${ }^{13} \mathrm{C}\left\{{ }^{1} \mathrm{H}\right\}$ NMR Spectrum of Ruthenium Complex 8 in $\mathrm{CD}_{2} \mathrm{Cl}_{2}$.
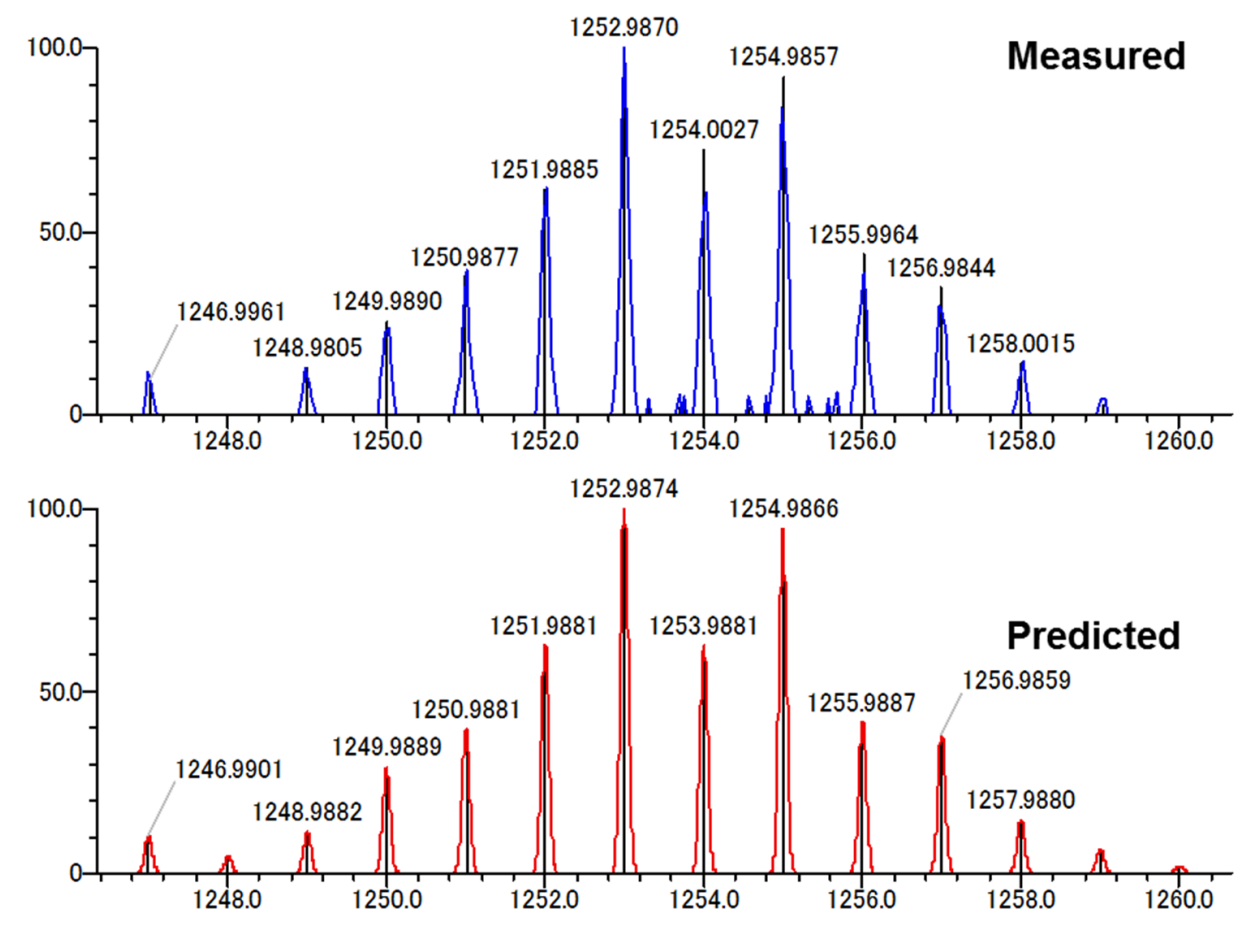

found $\mathrm{m} / \mathrm{z} 1252.9870$ [M] $^{+}$

calcd. for $\left[\mathrm{C}_{41} \mathrm{H}_{29} \mathrm{~N}_{3} \mathrm{~F}_{24} \mathrm{P}_{2} \mathrm{Cl}_{2} \mathrm{Ru}\right]^{+}$(1252.9874, $\left.-0.4 \mathrm{mDa},-0.32 \mathrm{ppm}\right)$

Figure S20. HRMS Spectrum of Ruthenium Complex 8. 

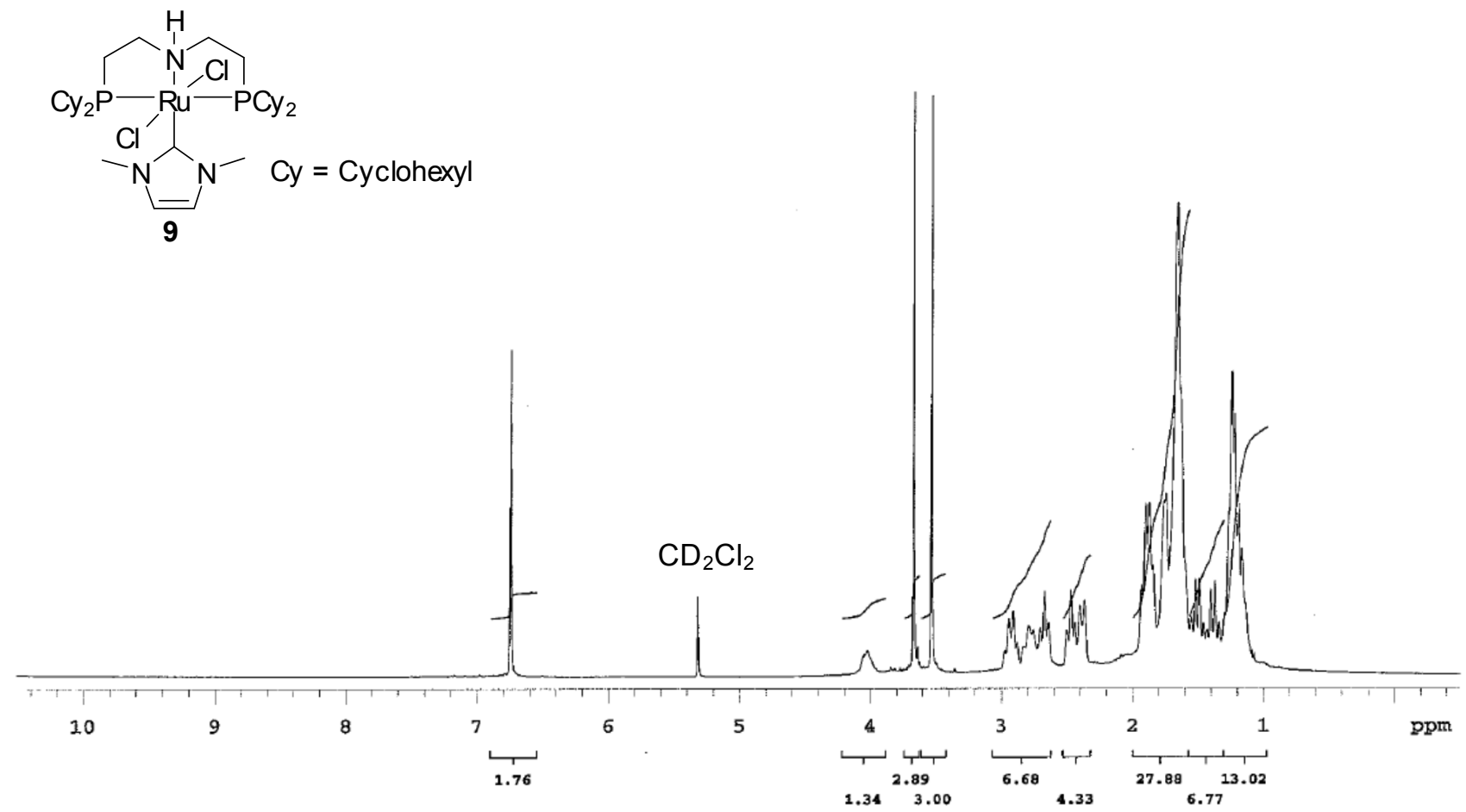

Figure S21. ${ }^{1} \mathrm{H}$ NMR Spectrum of Ruthenium Complex 9 in $\mathrm{CD}_{2} \mathrm{Cl}_{2}$.

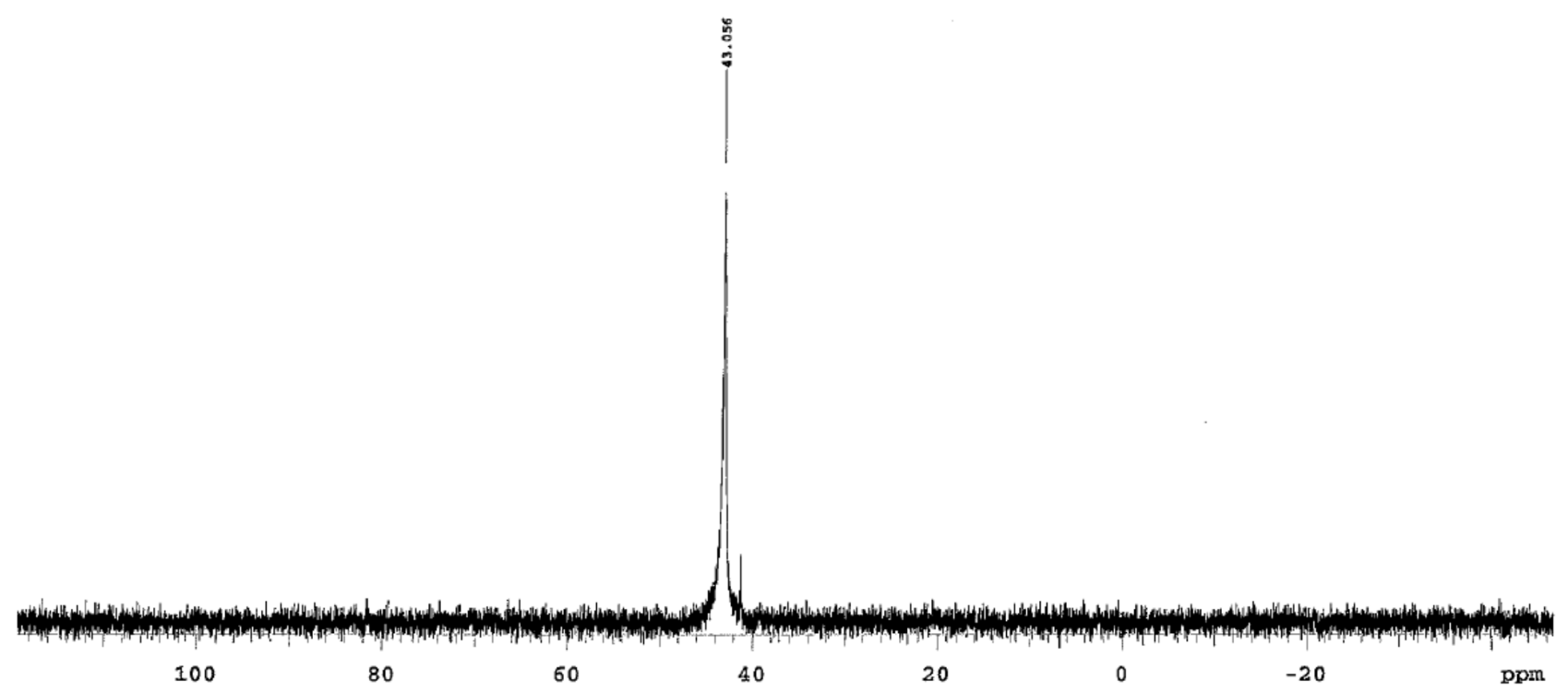

Figure S22. ${ }^{31} \mathrm{P}\left\{{ }^{1} \mathrm{H}\right\}$ NMR Spectrum of Ruthenium Complex 9 in $\mathrm{CD}_{2} \mathrm{Cl}_{2}$. 


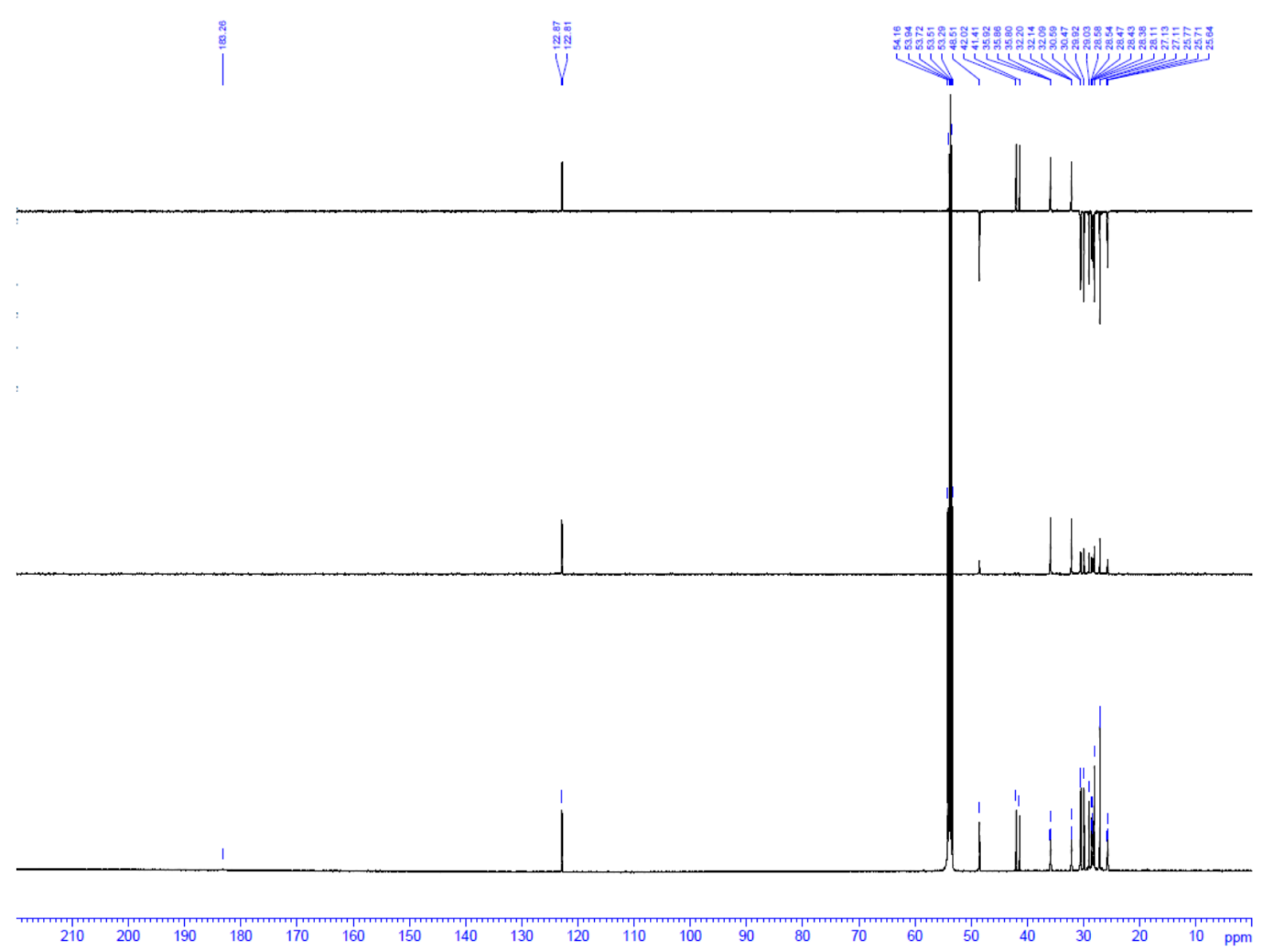

Figure S23. ${ }^{13} \mathrm{C}\left\{{ }^{1} \mathrm{H}\right\}$ NMR Spectrum of Ruthenium Complex 9 in $\mathrm{CD}_{2} \mathrm{Cl}_{2}$.

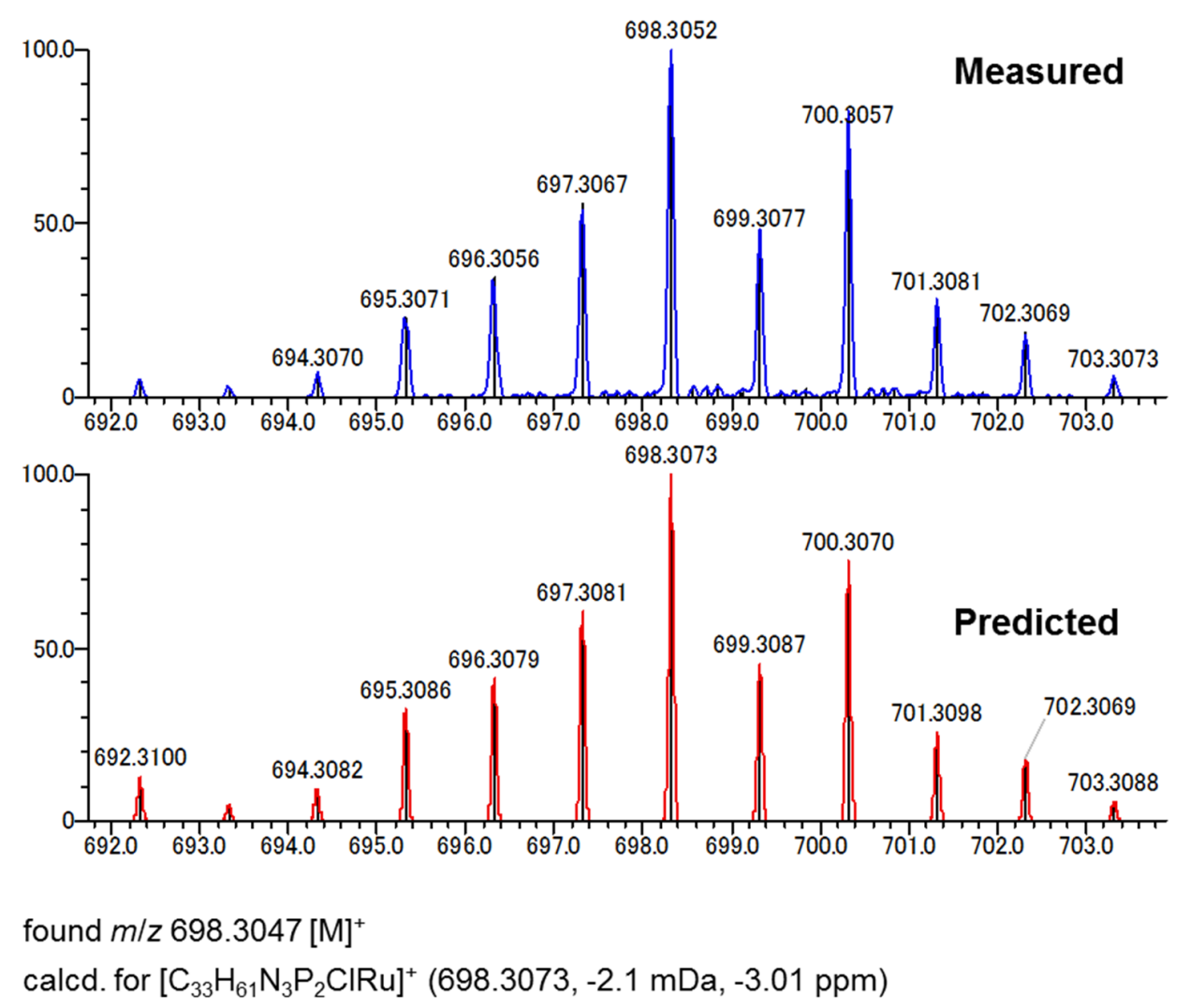

Figure S24. HRMS Spectrum of Ruthenium Complex 9. 


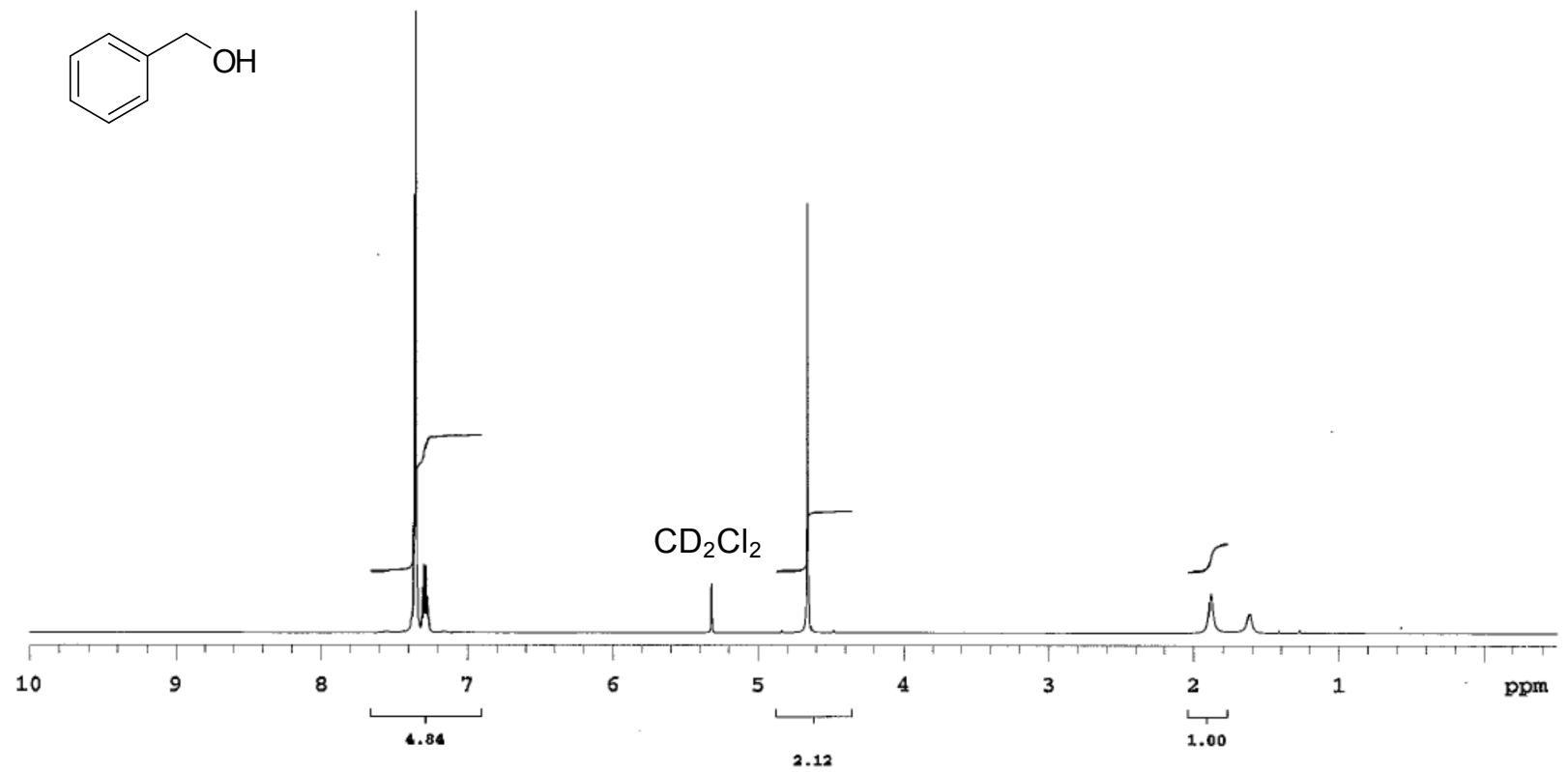

Figure S25. ${ }^{1} \mathrm{H}$ NMR Spectrum of Benzyl Alcohol in $\mathrm{CD}_{2} \mathrm{Cl}_{2}$.

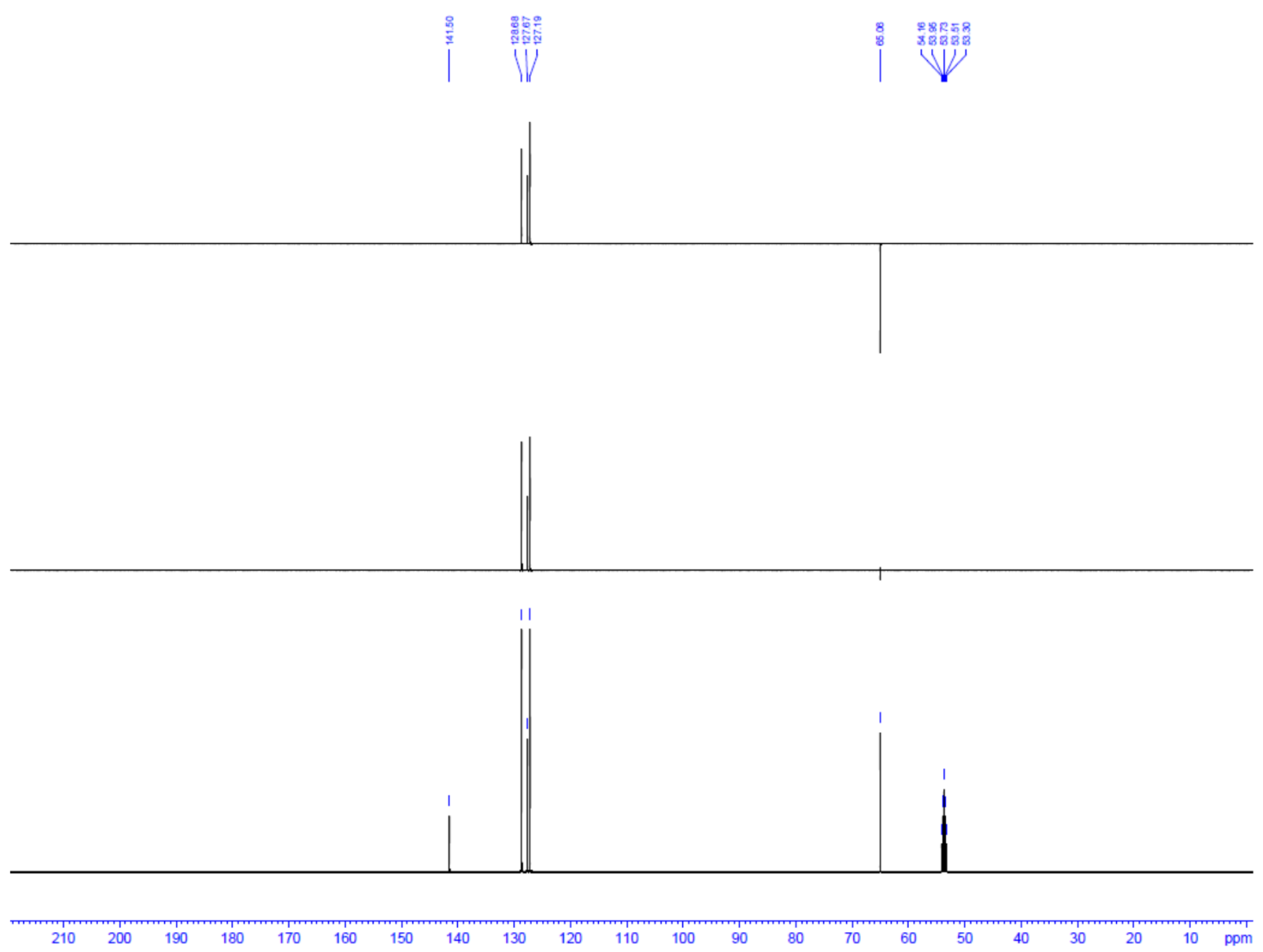

Figure S26. ${ }^{13} \mathrm{C}\left\{{ }^{1} \mathrm{H}\right\}$ NMR Spectrum of Methyl Benzoate in $\mathrm{CD}_{2} \mathrm{Cl}_{2}$. 


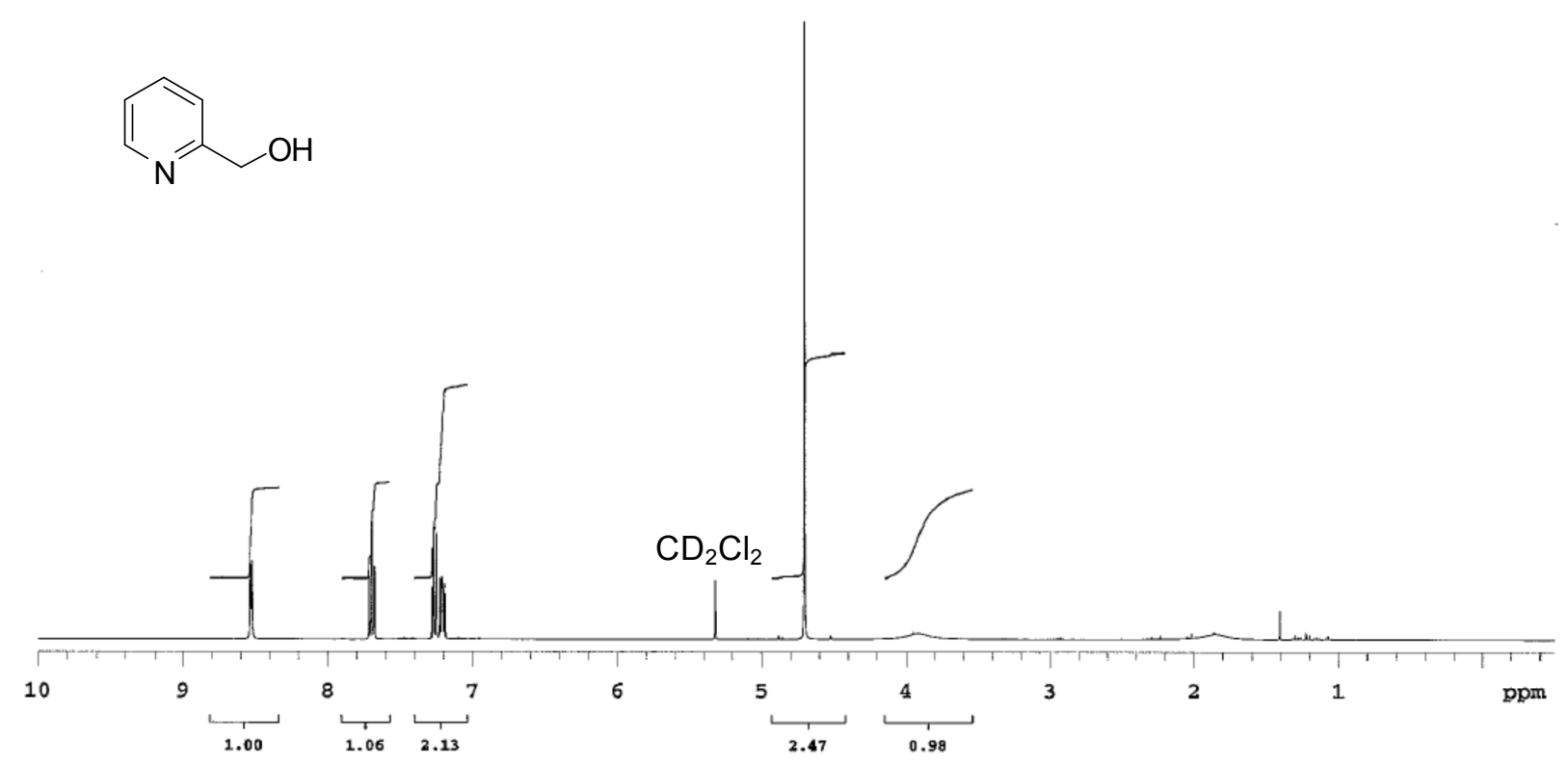

Figure S27. ${ }^{1} \mathrm{H}$ NMR Spectrum of 2-Pyridinemethanol in $\mathrm{CD}_{2} \mathrm{Cl}_{2}$.
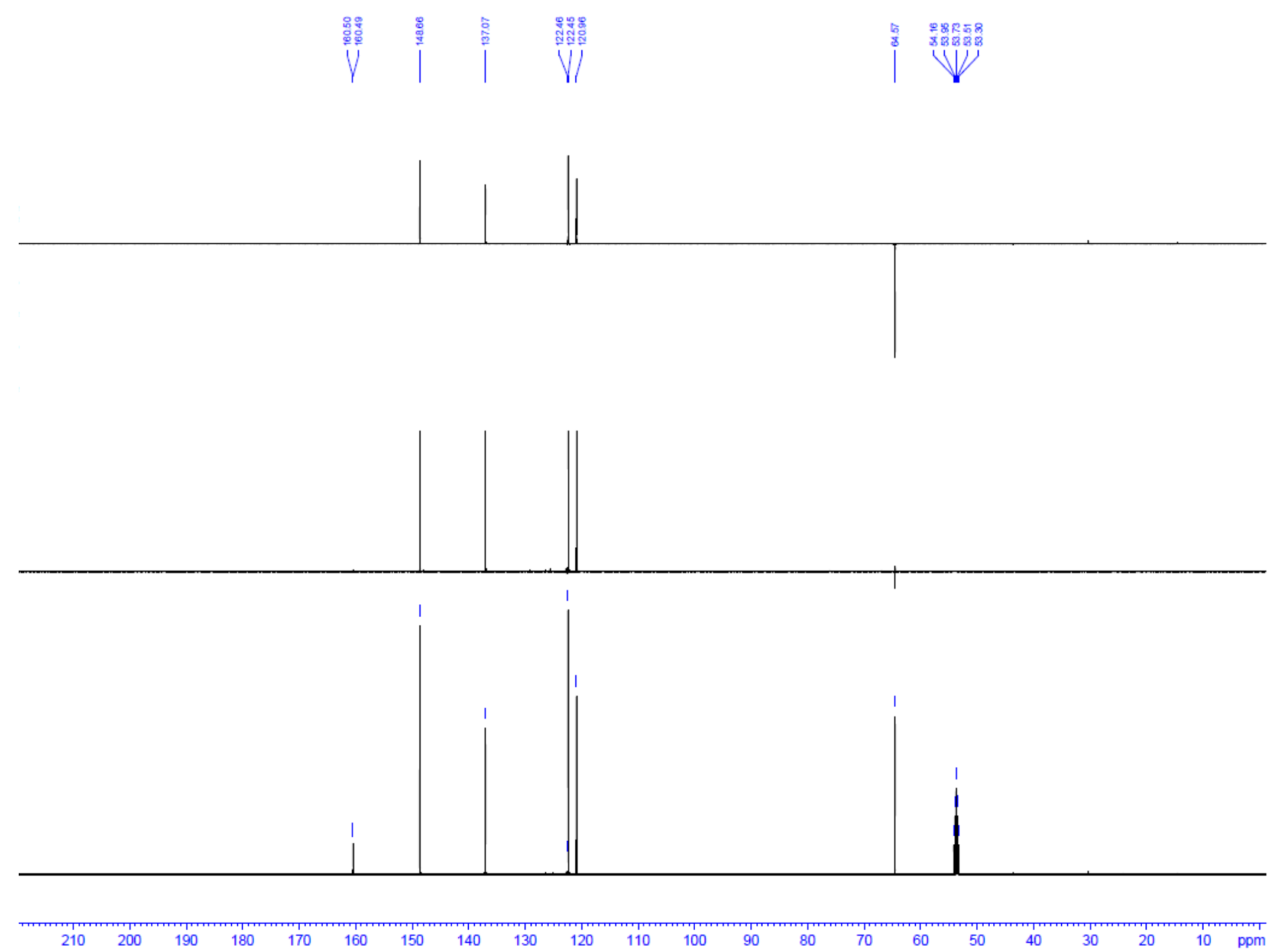

Figure S28. ${ }^{13} \mathrm{C}\left\{{ }^{1} \mathrm{H}\right\}$ NMR Spectrum of 2-Pyridinemethanol in $\mathrm{CD}_{2} \mathrm{Cl}_{2}$. 


\section{References}

1) (a) Lin, I. J. B.; Vasam, C. S. Coord. Chem. Rev. 2007, 251, 642. (b) Wang, H. M. J.; Lin, I. J. B. Organometallics 1998, 17, 972.

2) Holbrey, J. D.; Reichert, W. M.; Tkatchenko, I.; Bouajila, E.; Walter, O.; Tommasi, I.; Rogers, R. D. Chem. Commun. 2003, 28.

3) Roberts, G. M.; Pierce, P. J.; Woo, L. K. Organometallics 2013, 32, 2033.

4) Nuzzo, R. G.; Haynie, S. L.; Wilson, M. E.; Whitesides, G. M. J. Org. Chem. 1981, 46, 2861.

5) Danopoulos, A. A.; Willis, A. R.; Edwards, P. G. Polyhedron 1990, 9, 2413

6) Kuriyama, W.; Matsumoto, T.; Ogata, O.; Ino, Y.; Aoki, K.; Tanaka, S.; Ishida, K.; Kobayashi, T.; Sayo, N.; Saito, T. Org. Process Res. Dev. 2012, 16, 166.

7) a) Zhang, Y.; Chen, C.; Ghosh, S. C.; Li, Y.; Hong, S. H. Organometallics 2010, 29, 1374. (b) Voutchkova, A. M.; Appelhans, L. N.; Chianese, A. R.; Crabtree, R. H. J. Am. Chem. Soc. 2005, 127, 17624. 